\title{
Exergy-Based Optimal Control of a Vapor Compression System
}

\author{
Neera Jain $^{1 *}$, Andrew Alleyne ${ }^{2}$ \\ ${ }^{1}$ Purdue University \\ West Lafayette, IN, USA \\ neerajain@purdue.edu \\ ${ }^{2}$ University of Illinois at Urbana-Champaign \\ Urbana, IL, USA \\ alleyne@illinois.edu \\ *Corresponding Author
}

\begin{abstract}
Exergy-based analysis and optimization has been successfully used to design a variety of thermal systems to achieve greater efficiency. However, the advantages afforded by exergy destruction minimization (EDM) at the design stage have not been translated to closed-loop operation of thermal systems such as vapor compression systems (VCSs). Through online optimization and control, VCSs can effectively respond to disturbances, such as weather or varying loads that cannot be accounted for at the design stage, while simultaneously maximizing system efficiency. Furthermore, in applications where VCSs encounter high frequency disturbances, such as in refrigerated transport applications or passenger vehicles, optimizing efficiency at steady-state conditions alone may not lead to significant reductions in energy consumption. In this paper we design the first exergetic, or second law, optimal controller for a canonical four-component vapor compression system (VCS). A lumped parameter moving boundary modeling framework is used to model the two heat exchangers in the VCS. A model predictive controller is then designed and implemented in simulation using a dynamic exergy-based objective function to determine the optimal control actions for the VCS to maximize exergetic efficiency while achieving a desired cooling capacity. Simulation results show that an exergy-based model predictive controller minimizing exergy destruction achieves over $40 \%$ greater exergetic efficiency during operation than a comparable first law MPC. Moreover, the distribution of exergy destruction across individual components offers new insight into the effects of variable-speed actuators on system efficiency in VCSs.
\end{abstract}




\section{INTRODUCTION}

37 Exergy analysis has been used extensively in the thermodynamics community to understand the source of irreversibilities in a variety of thermal systems, thereby influencing design changes at the system or component level

39 [1]. In the context of vapor compression systems (VCSs), Ahamed et. al. [2] provide an extensive review of exergy

40

41

42

43

0 1 analyses that have been conducted, particularly highlighting the effect of different refrigerants, as well as key parameters such as evaporating temperature, on the exergetic efficiency of the system. Similarly, Padilla et. al. [3] use an exergy analysis to evaluate the use of R413A as an alternative refrigerant in an unmodified R12 vapor compression system. More recently, Mahabadipour and Ghaebi [4] used an exergy analysis to evaluate the better of two designs of expander cycles for refrigeration systems. In addition to exergy analyses, researchers have used exergy destruction minimization (EDM), also known as entropy generation minimization or thermodynamic optimization [5], to optimize design and operational parameters in many thermal systems from a static point of view. For example, design parameters such as heat exchanger geometry have been optimized using EDM by Nag and De [6] and Vargas and Bejan [7]. However, the advantages afforded by EDM at the design stage have not been translated to closed-loop operation of thermal systems, including VCSs. In this paper, we present the first use of transient exergy destruction as a metric for a closed-loop decision-making algorithm.

In order to meet the increasing demand for more efficient VCSs, effective control of these systems is required. Through online optimization and control, VCSs can effectively respond to disturbances such as changes in ambient conditions or time-varying thermal loads that cannot be accounted for at the design stage alone. Techniques from optimal control theory [8] can be used to design system controllers that optimally balance competing objectives such as performance and efficiency. However, to do so requires a mathematical characterization of these quantities. Therefore, the goal of this paper is to enable the use of EDM for feedback control of VCSs by first modeling the transient effects of changes in control variables on the rate of exergy destruction in a canonical VCS and then using this model for the design and implementation of an exergy-based model predictive controller. 


\section{BACKGROUND}

\subsection{Exergy}

Exergy (also referred to as "availability") is defined as the maximum reversible work that can be extracted from a substance at a given state during its interaction with a given environment [9]. Whereas energy is always conserved, exergy is not. Similarly to energy, exergy can be transferred in three ways: by heat transfer, work, or through mass exchange with the environment. However, contrary to energy, exergy is destroyed during irreversible phenomena such as chemical reactions, mixing, and viscous dissipation. The rate of change of exergy in a control volume is defined mathematically as

$$
\frac{d X_{c v}}{d t}=\frac{d E_{c v}}{d t}-T_{0} \frac{d S_{c v}}{d t}=\sum_{j}\left(1-\frac{T_{0}}{T_{j}}\right) \dot{Q}_{j}-\left(\dot{W}_{c v}-P_{0} \frac{d V_{c v}}{d t}\right)+\sum_{i} \dot{m}_{i} \psi_{i}-\sum_{o} \dot{m}_{o} \psi_{o}-\dot{X}_{d e s t},
$$

where $\dot{Q}$ is the heat transfer rate at the location on the control volume boundary where the instantaneous temperature is $T_{j}[10]$. The specific flow exergy, $\psi$, is defined as

$$
\psi=\left(h-h_{0}\right)-T_{0}\left(s-s_{0}\right)+\frac{v^{2}}{2}+g z+\psi^{\mathrm{ch}}
$$

where the quantities $T_{0}, P_{0}, h_{0}$, and $s_{0}$ are the temperature, pressure, specific enthalpy, and specific entropy, respectively, of the reference environment. The reference environment is typically chosen as an infinite reservoir with which the system is interacting, such as the ambient environment. The amount of exergy destroyed in a system or through a process is a measure of the loss of potential to do work and is proportional to the amount of entropy generated in the system as stated by the Gouy-Stodola theorem shown in Eq. (3) [11].

$$
\dot{X}_{d e s t}=T_{0} \dot{S}_{g e n}
$$

The most common efficiency metric used for VCSs is the coefficient of performance (COP), defined as the ratio of the heat energy removed to the energy consumed (by the VCS). This quantity is generally greater than one. However, the maximum achievable COP is limited by the temperatures of the hot and cold environments that interact with the VCS [12]. Therefore, it is more informative to consider the second law, or exergetic, efficiency, $\eta_{\mathrm{II}}$, which characterizes efficiency relative to the maximum achievable efficiency as postulated by the second law of thermodynamics: 


$$
0 \leq \eta_{\mathrm{II}}=\left\{1-\frac{\text { exergy destroyed }}{\text { exergy supplied }}\right\}<1
$$

Equation (4) states that minimizing exergy destroyed in a system will maximize its exergetic efficiency.

\subsection{Reversible Work}

In the context of optimization of thermal energy systems, it is common for the amount of power consumed (or generated) in a system to be minimized (or maximized). The relationship between the minimization of exergy destruction rate versus the minimization of power consumption is characterized by

$$
\dot{X}_{\text {dest }}=\dot{W}-\dot{W}_{\text {rev }}
$$

and is equivalent to Eq. (3) [5]. The reversible rate of change of work, $\dot{W}_{\text {rev }}$, also called reversible power, refers to the amount of power that the system would consume (or produce) if there were no irreversibilities in the system. Let us define two objective functions, $J_{1}$ and $J_{2}$, where $J_{1}$ is equal to the rate of exergy destruction and $J_{2}$ is equal to the actual power consumed (or produced).

$$
J_{1}=\dot{X}_{d e s t}=\dot{W}-\dot{W}_{\text {rev }}
$$

$$
J_{2}=\dot{W}
$$

Equation (5) implies that if the quantity $\dot{W}_{\text {rev }}$ is constant with respect to the decision variables of an optimization problem, then the minimizations of $J_{1}$ and $J_{2}$ will be equivalent as shown in Eq. (8) [5]. That is to say, the minimization of exergy destruction rate and minimization of power will be equivalent.

$$
\min _{W_{r e v}=C}\left(J_{1}\right)=\min \left(J_{2}\right)
$$

In Section 5 we will specifically present a case study when this equivalence does not exist and discuss its impact on closed-loop control of a VCS.

\subsection{VCS Modeling and Control}

For dynamic modeling of heat exchangers, two different approaches have been primarily used: a finite-volume (FV) approach and a lumped parameter moving boundary (LPMB) approach [13]. In the LPMB modeling approach, the heat exchanger is modeled with a fixed number of fluid regions (defined by fluid phase), and the location of the boundary between each fluid region is a dynamic variable, allowing the length of the fluid regions to vary. Fluid 
properties such as temperature, density, etc., are lumped in each region, and an average is used for model computations. Although this approach results in some loss in accuracy as compared to a FV approach, the resulting models are of low dynamic order, making them well suited for control design. A review of the literature shows that the LPMB approach has been applied to a variety of VCSs, often with variations in the details of the modeling approach [14]. The LPMB condenser and evaporator models that are used as the basis for the derivation of the transient exergy destruction rate in this paper are described in detail in [15] and [16].

120

The control of vapor compression systems (VCSs) varies depending upon the available hardware. For VCSs with only a fixed-speed compressor and condenser fan, on-off control prevails; typically a mechanically-controlled thermostatic expansion valve regulates evaporator superheat and users are given manual control over the evaporator fan speed. Commercial variable-speed VCSs are typically controlled using multiple proportional-integral (PI) controllers coupled with discrete logic to handle critical constraints and transitions between operation modes [17]. However, a range of advanced control methodologies than can better mitigate the multivariable nature of VCSs have been proposed in the literature, including [18] [19] [20] [21] [22]. A major focus of these methodologies has been achieving desired performance objectives (e.g. room temperature regulation) while minimizing energy consumption or maximizing COP [23] [24] [25]. However, as discussed in Section 2.1, COP is limiting in its ability to characterize the true efficiency of a VCS. Therefore, we are interested in studying exergy destruction minimization as an alternative metric for controlling vapor compression systems for maximum exergetic efficiency. For the current work, Model Predictive Control [26] [27] was chosen as an approach that gives a fair comparison between multiple objective functions being used to optimize the same system.

\section{6}

\subsection{Derivation of Transient Exergy Destruction Rate}

137 Here we consider a canonical four-component VCS (Figure 1a). Additional components such as receiver, oil 138 separator, etc. are not included to simplify this first investigation of EDM for real-time control. To develop a 139 transient (dynamic) expression for the total rate of exergy destruction in the VCS, it is necessary to consider each 140 component individually as a control volume as shown in Figure $1 \mathrm{~b}$ which demonstrates the control volumes 141 encompassing solely the refrigerant. 


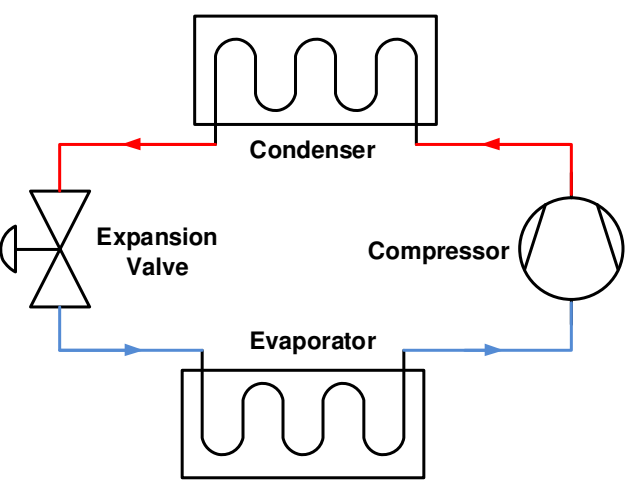

a.

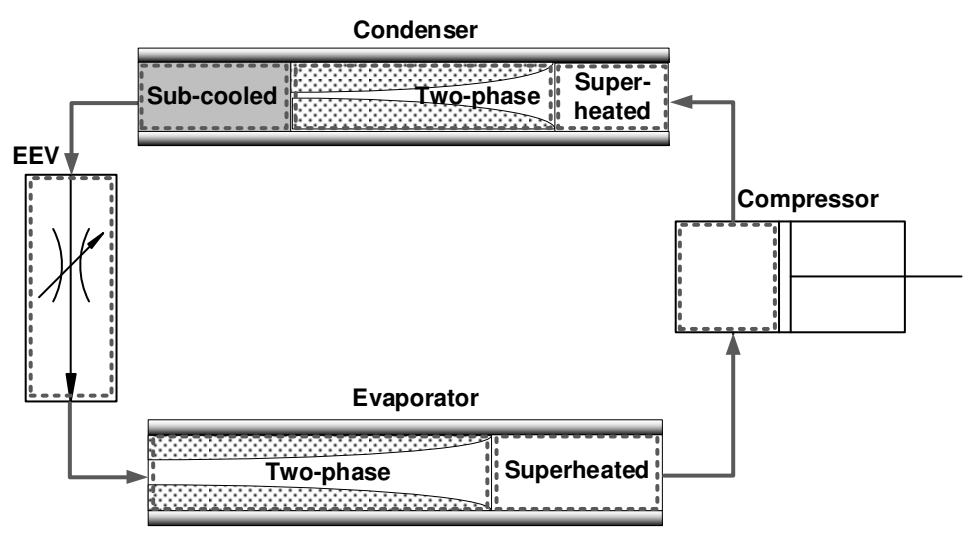

b.
142

Figure 1: a. VCS schematic; b. VCS schematic depicting control volumes drawn inside each component.

The total rate of exergy destruction in a canonical VCS is a sum of the rates of exergy destruction in each individual component:

$$
\dot{X}_{d e s t, V C S}=\dot{X}_{d e s t, k}+\dot{X}_{d e s t, v}+\dot{X}_{d e s t, c}+\dot{X}_{d e s t, e} .
$$

In the following sections, the exergy destruction rate for each component will be derived [28]. Note that evaporator and condenser fans are not considered in this analysis for the purpose of illustrative clarity. They could be added if needed, but the refrigerant-focused construct here is sufficient for characterizing the transient exergy destruction rate in the VCS. The reference temperature, $T_{0}$, for the exergy calculation is assumed to be the temperature of the hightemperature reservoir (i.e. ambient environment), $T_{H}$, with which the VCS interacts.

\subsubsection{Compressor and EEV}

In VCS modeling, both the compressor and expansion device, assumed here to be an electronic expansion valve (EEV), are typically modeled using quasi-steady assumptions. Therefore, the compressor and EEV control volumes can be analyzed assuming steady-state operation. The compressor is assumed to be adiabatic but not isentropic; therefore, there is no exergy transfer by heat transfer. A control volume is defined around the refrigerant inside the compressor as shown in Figure 1b. The inlet and outlet mass flow rates are equal to the refrigerant mass flow rate through the compressor. Assuming steady state operation, Eq. (1) reduces to 


$$
-\left(-\dot{W}_{k}\right)+\dot{m}_{r, k}\left(\psi_{k, i}-\psi_{k, o}\right)-\dot{X}_{d e s t, k}=0
$$

161 The effects of kinetic and potential energy are assumed to be negligible. Substituting Eq. (2) into Eq. (10) and 162 simplifying yields

$$
\dot{X}_{\text {dest }, k}=-\left(-\dot{W}_{k}\right)+\dot{m}_{r, k}\left[\left(h_{k, r i}-h_{k, r o}\right)-T_{H}\left(s_{k, r i}-s_{k, r o}\right)\right] .
$$

164 Note that the work transfer rate term in Eq. (11) must be a positive quantity because if the compressor was 165 isentropic, then the rate of exergy destruction would equal zero (and $h_{k, r i}-h_{k, r o}$ is a negative quantity). Therefore,

$-\left(-\dot{W}_{k}\right)$ to emphasize the fact that the sign convention for work done on the system is negative, where

$$
\dot{W}_{k}=\dot{m}_{r, k}\left(h_{k, r o}-h_{k, r i}\right) \text {. }
$$

The rate of exergy destruction in the compressor is determined by substituting Eq. (12) into Eq. (11) and 170 simplifying:

$$
\dot{X}_{d e s t, k}=-T_{H} \dot{m}_{r, k}\left(s_{k, r i}-s_{k, r o}\right) .
$$

172 To derive the rate of exergy destruction in the EEV, a control volume is defined around the refrigerant in the EEV.

173 The expansion of the refrigerant is assumed to be isenthalpic (i.e. $h_{v, r i}=h_{v, r o}$ ). There is only exergy transfer by mass transfer, and the inlet and outlet mass flow rates are equal to the refrigerant mass flow rate through the EEV.

\section{5} refrigerant fluid region. In this way, separate lumped parameters are used to estimate the fluid properties in each of Assuming steady-state operation and regarding the effects of kinetic and potential energy as negligible gives

$$
\dot{X}_{\text {dest }, v}=-T_{H} \dot{m}_{r, v}\left(s_{v, r i}-s_{v, r o}\right) .
$$

\subsubsection{Heat Exchangers}

The remaining components in the current VCS model are the two heat exchangers: the evaporator and the condenser. The dynamics of these components dominate the overall dynamics of the cycle; consequently, transient rates of exergy destruction through each of these components will be derived. In the LPMB modeling approach, the evaporator is typically modeled with two fluid regions: a two-phase refrigerant fluid region and a superheated the fluid regions, thereby improving the accuracy of the estimates. Similarly, we use two separate control volumes to derive the total exergy destruction rate through the evaporator as shown in Figure 2. 
186

187

188

191

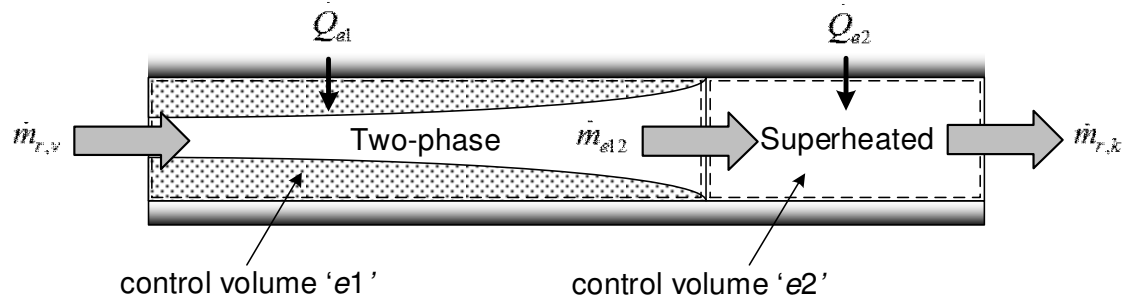

Figure 2: Individual control volumes drawn around each fluid region in an evaporator.

For the two-phase refrigerant fluid region, denoted by the subscript $e$, Eq. (1) reduces to

$$
\frac{d X_{e 1}}{d t}=\left(1-\frac{T_{H}}{T_{w, e 1}}\right) \dot{Q}_{e 1}+P_{0} \frac{d V_{e 1}}{d t}+\dot{m}_{r, v}\left(h_{e, r i}-T_{H} s_{e, r i}\right)-\dot{m}_{e 12}\left(h_{e, g}-T_{H} s_{e, g}\right)-\dot{X}_{d e s t, e 1},
$$

where $T_{j}$ is replaced with $T_{w, e 1}$, the lumped tube wall temperature in the two-phase fluid region, and $\dot{m}_{e 12}$ is the refrigerant mass flow rate between the two control volumes pictured in Figure 2. Similarly, for the superheated refrigerant fluid region, denoted by the subscript $e 2$, Eq. (1) reduces to

$$
\frac{d X_{e 2}}{d t}=\left(1-\frac{T_{H}}{T_{w, e 2}}\right) \dot{Q}_{e 2}+P_{0} \frac{d V_{e 2}}{d t}+\dot{m}_{e 12}\left(h_{e, g}-T_{H} s_{g}\right)-\dot{m}_{r, k}\left(h_{e, r o}-T_{H} s_{e, r o}\right)-\dot{X}_{d e s t, e 2},
$$

where $T_{j}$ is replaced with $T_{w, e 2}$, the lumped tube wall temperature in the superheated fluid region. Applying superposition allows us to express $\dot{X}_{\text {dest }, e}$ as

$$
\dot{X}_{d e s t, e}=\dot{X}_{d e s t, e 1}+\dot{X}_{d e s t, e 2} \text {. }
$$

Therefore, the total exergy destruction rate through the evaporator is

$$
\begin{aligned}
\dot{X}_{\text {dest }, e} & =\left(1-\frac{T_{H}}{T_{w, e 1}}\right) \dot{Q}_{e 1}+\left(1-\frac{T_{H}}{T_{w, e 2}}\right) \dot{Q}_{e 2}+P_{0}\left(\frac{d V_{e 1}}{d t}+\frac{d V_{e 2}}{d t}\right)+\dot{m}_{r, v}\left(h_{e, r i}-T_{H} s_{e, r i}\right) \\
& -\dot{m}_{r, k}\left(h_{e, r o}-T_{H} s_{e, r o}\right)-\left(\frac{d X_{e 1}}{d t}+\frac{d X_{e 2}}{d t}\right) .
\end{aligned}
$$

where it is assumed that

$$
\dot{Q}_{e}=\dot{Q}_{e 1}+\dot{Q}_{e 2}=(U A)_{e 1}\left(T_{w, e 1}-T_{r, e 1}\right)+(U A)_{e 2}\left(T_{w, e 2}-T_{r, e 2}\right) .
$$

In other words, it is assumed that there is no heat transfer between the refrigerant in control volume $e 1$ and the refrigerant in control volume $e 2$. In Eq. (19), $T_{r, e 1}$ and $T_{r, e 2}$ refer to the lumped refrigerant temperatures in each fluid region, and $(U A)_{e 1}$ and $(U A)_{e 2}$ are the overall heat transfer coefficients between the refrigerant and tube wall in each fluid region. 
206 An alternative method for deriving the exergy destruction rate is to perform an entropy balance on the control 207 volume using Eq. (20), solve for the rate of entropy generation $\dot{S}_{g e n}$, and then scale $\dot{S}_{g e n}$ by the reference environment 208 temperature, $T_{H}$.

$$
\frac{d S_{c v}}{d t}=\sum_{j} \frac{\dot{Q}_{j}}{T_{j}}+\sum_{i} \dot{m}_{i} s_{i}-\sum_{o} \dot{m}_{o} s_{o}+\dot{S}_{g e n}
$$

Because it is difficult to evaluate $d X_{c v} / d t$, the entropy rate balance given in Eq. (20) can be used to derive an

211 expression for the exergy destruction rate in terms of $d S_{c v} / d t$ instead of $d X_{c v} / d t$. Applying Eq. (20) to each 212 control volume of the evaporator yields

where

$$
\frac{d S_{e 1}}{d t}=\frac{\dot{Q}_{e 1}}{T_{w, e 1}}+\left(\dot{m}_{r, v} S_{e, r i}-\dot{m}_{e 12} s_{e, g}\right)+\dot{S}_{g e n, e 1},
$$

$$
\frac{d S_{e 2}}{d t}=\frac{\dot{Q}_{e 2}}{T_{w, e 2}}+\left(\dot{m}_{e 12} S_{e, g}-\dot{m}_{r, k} s_{e, r o}\right)+\dot{S}_{g e n, e 2}
$$

Substituting Eq. (21) and Eq. (22) into Eq. (23), rearranging terms, and scaling by $T_{H}$ yields the following alternative expression for the exergy destruction rate in the evaporator:

$$
\dot{X}_{d e s t, e}=T_{H} \dot{S}_{g e n, e}=-T_{H}\left(\frac{\dot{Q}_{e 1}}{T_{w, e 1}}+\frac{\dot{Q}_{e 2}}{T_{w, e 2}}\right)-\left(\dot{m}_{r, v} T_{H} s_{e, r i}-\dot{m}_{r, k} T_{H} s_{e, r o}\right)+T_{H}\left(\frac{d S_{e 1}}{d t}+\frac{d S_{e 2}}{d t}\right)
$$

In the LPMB modeling framework, the condenser is typically modeled with three refrigerant fluid regions: a superheated fluid region, a two-phase fluid region, and a subcooled fluid region. To remain consistent with this modeling approach, three separate control volumes are used to derive the total exergy destruction rate through the condenser as shown in Figure 3. Although at steady-state it can be assumed that the heat transfer out of the condenser is occurring at the reference temperature, $T_{H}$, the control volumes defined in Figure 3 for the condenser only contain the refrigerant flowing through the condenser tube. Therefore, the transfer of heat away from the 
refrigerant is occurring at the tube wall temperatures of each fluid region. The procedure for deriving the total exergy destruction rate through the condenser is analogous to the procedure described for the evaporator, and so we present only the final result in Eq. (25),

which is equivalent to

$$
\begin{aligned}
\dot{X}_{d e s t, c} & =\left(1-\frac{T_{H}}{T_{w, c 1}}\right)\left(-\dot{Q}_{c 1}\right)+\left(1-\frac{T_{H}}{T_{w, c 2}}\right)\left(-\dot{Q}_{c 2}\right)+\left(1-\frac{T_{H}}{T_{w, c 3}}\right)\left(-\dot{Q}_{c 3}\right)+P_{0}\left(\frac{d V_{c 1}}{d t}+\frac{d V_{c 2}}{d t}+\frac{d V_{c 3}}{d t}\right) \\
& +\dot{m}_{r, k}\left(h_{c, r i}-T_{H} s_{c, r i}\right)-\dot{m}_{r, v}\left(h_{c, r o}-T_{H} s_{c, r o}\right)-\left(\frac{d X_{c 1}}{d t}+\frac{d X_{c 2}}{d t}+\frac{d X_{c 3}}{d t}\right)
\end{aligned}
$$

Figure 3: Individual control volumes drawn around each fluid region in a condenser.

\subsubsection{The Entropy Differential}

The rate of change of entropy can be described as [29]

$$
\frac{d S_{c v}}{d t}=s_{c v} \frac{d m_{c v}}{d t}+m_{c v}\left(\left.\frac{\left(\partial s_{c v}\right)}{\partial h}\right|_{P} \frac{d h}{d t}+\left.\frac{\left(\partial s_{c v}\right)}{\partial P}\right|_{h} \frac{d P}{d t}\right) .
$$

In Eq. (27) the dependent variables are chosen as specific enthalpy and pressure, but they can be chosen as any two independent thermodynamic state variables. As was mentioned in Section 2.3, the LPMB modeling approach is desirable from a controls perspective because of the low dynamic order of the resulting model. However, the consequence of a low order model is that only certain time derivatives are defined. Therefore, the ability to reexpress Eq. (27) using different thermodynamic state variables is necessary. Equation (27) also highlights why it is helpful to define multiple control volumes for the heat exchangers in which a separate control volume is drawn 
approximate $s_{c v}$ and $m_{c v}$ for each control volume as is done in the LPMB framework. The expression for $d m_{c v} / d t$ can be derived for each control volume as described in [30].

251 The dynamic state variables for the evaporator are $\mathbf{x}_{e}=\left[\begin{array}{llllll}\zeta_{e 1} & P_{e} & h_{e 2} & T_{w, e 1} & T_{w, e 2} & \bar{\gamma}_{e}\end{array}\right]^{T}$. For the two-phase fluid region of the evaporator, denoted by the subscript $e 1$, the time derivative of enthalpy is not available. Instead, refrigerant mean void fraction, $\bar{\gamma}_{e}$, and pressure, $P_{e}$, can be used to describe specific entropy for the two-phase fluid region, as shown in Eq. (28), where $m_{e 1}=\rho_{e 1} \zeta_{e 1} L_{R, e} A_{C R, e}$.

The rate of change of mass in the control volume is given by

$$
\frac{d S_{e 1}}{d t}=s_{e 1} \frac{d m_{e 1}}{d t}+m_{e 1}\left(\left.\frac{\left(\partial s_{e 1}\right)}{\partial \bar{\gamma}_{e}}\right|_{P} \frac{d \bar{\gamma}_{e}}{d t}+\left.\frac{\left(\partial s_{e 1}\right)}{\partial P_{e}}\right|_{\bar{\gamma}} \frac{d P_{e}}{d t}\right)
$$

and the specific entropy in the control volume is given by

$$
\frac{d m_{e 1}}{d t}=\left(\dot{m}_{r, v}-\dot{m}_{e 12}\right)+\rho_{e 1} A_{C R, e} L_{R, e} \frac{d \zeta_{e 1}}{d t}
$$

Mean void fraction, $\bar{\gamma}$, is related to mean quality, $\bar{x}$, by the following relationship:

$$
s_{e 1}=\bar{x}_{e} s_{e, g}+\left(1-\bar{x}_{e}\right) s_{e, l}=\frac{\bar{\gamma}_{e} \rho_{e, g} s_{e, g}+\left(1-\bar{\gamma}_{e}\right) \rho_{e, l} s_{e, l}}{\bar{\gamma}_{e} \rho_{e, g}+\left(1-\bar{\gamma}_{e}\right) \rho_{e, l}}
$$

$$
\bar{x}=\bar{\gamma} \frac{\rho_{g}}{\rho} .
$$

262 The variables $\rho_{e, l}, \rho_{e, g}, s_{e, l}$, and $s_{e, g}$ are all solely functions of pressure. The partial derivatives $\left.\frac{\partial s_{e 1}}{\partial \bar{\gamma}_{e}}\right|_{P}$ and $\left.\frac{\partial s_{e 1}}{\partial P_{e}}\right|_{\bar{\gamma}}$, shown in Eq. (32) and Eq. (33) respectively, are derived using Eq. (30).

$$
\left.\frac{\partial s_{e 1}}{\partial \bar{\gamma}_{e}}\right|_{P}=\frac{\left(\bar{\gamma}_{e} \rho_{e, g}+\left(1-\bar{\gamma}_{e}\right) \rho_{e, l}\right)\left(\rho_{e, g} s_{e, g}-\rho_{e, l} s_{e, l}\right)-\left(\bar{\gamma}_{e} \rho_{e, g} s_{e, g}+\left(1-\bar{\gamma}_{e}\right) \rho_{e, l} s_{e, l}\right)\left(\rho_{e, g}-\rho_{e, l}\right)}{\left(\bar{\gamma}_{e} \rho_{e, g}+\left(1-\bar{\gamma}_{e}\right) \rho_{e, l}\right)^{2}}
$$




$$
\begin{aligned}
\left.\frac{\partial s_{e 1}}{\partial P_{e}}\right|_{\bar{\gamma}} & =\frac{\beta_{1}-\beta_{2}}{\beta_{3}} \\
\beta_{1} & =\left(\bar{\gamma}_{e} \rho_{e, g}+\left(1-\bar{\gamma}_{e}\right) \rho_{e, l}\right)\left(\bar{\gamma}_{e} \rho_{e, g} \frac{d s_{e, g}}{d P_{e}}+s_{e, g} \bar{\gamma}_{e} \frac{d \rho_{e, g}}{d P_{e}}+\left(1-\bar{\gamma}_{e}\right) \rho_{e, l} \frac{d s_{e, l}}{d P_{e}}+s_{e, l}\left(1-\bar{\gamma}_{e}\right) \frac{d \rho_{e, l}}{d P_{e}}\right) \\
\beta_{2} & =\left(\bar{\gamma}_{e} \rho_{e, g} s_{e, g}+\left(1-\bar{\gamma}_{e}\right) \rho_{e, l} s_{e, l}\right)\left(\bar{\gamma}_{e} \frac{d \rho_{e, g}}{d P_{e}}+\left(1-\bar{\gamma}_{e}\right) \frac{d \rho_{e, l}}{d P_{e}}\right) \\
\beta_{3} & =\left(\bar{\gamma}_{e} \rho_{e, g}+\left(1-\bar{\gamma}_{e}\right) \rho_{e, l}\right)^{2}
\end{aligned}
$$

For the superheated (single-phase) fluid region in the evaporator, the time derivative of the lumped enthalpy, $h_{e 2}$, is defined as a dynamic state. Therefore, Eq. (27) is used to describe the rate of change of entropy in the superheated fluid region of the evaporator, yielding

$$
\frac{d S_{e 2}}{d t}=s_{e 2} \frac{d m_{e 2}}{d t}+\rho_{e 2} \zeta_{e 2} L_{R, e} A_{C R, e}\left(\left.\frac{\left(\partial s_{e 2}\right)}{\partial h_{e 2}}\right|_{P} \frac{d h_{e 2}}{d t}+\left.\frac{\left(\partial s_{e 2}\right)}{\partial P_{e}}\right|_{h} \frac{d P_{e}}{d t}\right)
$$

272 where

$$
\frac{d m_{e 2}}{d t}=\left(\dot{m}_{e 12}-\dot{m}_{r, k}\right)+\rho_{g, e} A_{C R, e} L_{R, e} \frac{d \zeta_{e 1}}{d t}
$$
the condenser control volumes: $d S_{c i} / d t, i=\{1,2,3\}$. It is assumed that the outlet refrigerant condition of the condenser is subcooled liquid; therefore, the condenser is characterized using three fluid regions. As in the case of the evaporator, specific enthalpy and pressure are used to describe the rate of change of specific entropy in the single-phase (superheated and subcooled) fluid regions, and mean void fraction and pressure are used in the twophase fluid region. The expressions for $d S_{c i} / d t, i=\{1,2,3\}$ are given in Eqs. (36) - (41), respectively. 


$$
\frac{d S_{c 1}}{d t}=s_{c 1} \frac{d m_{c 1}}{d t}+\rho_{c 1} \zeta_{c 1} L_{R, c} A_{C R, c}\left(\left.\frac{\left(\partial s_{c 1}\right)}{\partial h_{c 1}}\right|_{P} \frac{d h_{c 1}}{d t}+\left.\frac{\left(\partial s_{c 1}\right)}{\partial P_{c}}\right|_{h} \frac{d P_{c}}{d t}\right)
$$

$$
\frac{d m_{c 1}}{d t}=\left(\dot{m}_{r, k}-\dot{m}_{c 12}\right)+\rho_{g, c} A_{C R, c} L_{R, c} \frac{d \zeta_{c 1}}{d t}
$$

$$
\frac{d S_{c 2}}{d t}=s_{c 2} \frac{d m_{c 2}}{d t}+\rho_{c 2} \zeta_{c 2} L_{R, c} A_{C R, c}\left(\left.\frac{\left(\partial s_{c, 2}\right)}{\partial \bar{\gamma}_{c}}\right|_{P} \frac{d \bar{\gamma}_{c}}{d t}+\left.\frac{\left(\partial s_{c, 2}\right)}{\partial P_{c}}\right|_{\bar{\gamma}} \frac{d P_{c}}{d t}\right)
$$

$$
\frac{d m_{c 2}}{d t}=\left(\dot{m}_{c 12}-\dot{m}_{c 23}\right)+\rho_{l, c} A_{C R, c} L_{R, c}\left(\frac{d \zeta_{c 1}}{d t}+\frac{d \zeta_{c 2}}{d t}\right)-\rho_{g, c} A_{C R, c} L_{R, c} \frac{d \zeta_{c 1}}{d t}
$$

$$
\frac{d S_{c 3}}{d t}=s_{c 3} \frac{d m_{c 3}}{d t}+\rho_{c 3} \zeta_{c 3} L_{R, c} A_{C R, c}\left(\left.\frac{\left(\partial s_{c 3}\right)}{\partial h_{c 3}}\right|_{P} \frac{d h_{c 3}}{d t}+\left.\frac{\left(\partial s_{c 3}\right)}{\partial P_{c}}\right|_{h} \frac{d P_{c}}{d t}\right)
$$

$$
\frac{d m_{c 3}}{d t}=\left(\dot{m}_{c 23}-\dot{m}_{r, v}\right)-\rho_{l, c} A_{C R, c} L_{R, c}\left(\frac{d \zeta_{c 1}}{d t}+\frac{d \zeta_{c 2}}{d t}\right)
$$

Finally, substituting Eqs. (13), (14), (24), and (26) into Eq. (9) and simplifying results in the following expression for the total instantaneous exergy destruction rate in the VCS:

$$
\dot{X}_{d e s t, V C S}=T_{H}\left(\frac{\dot{Q}_{c 1}}{T_{w, c 1}}+\frac{\dot{Q}_{c 2}}{T_{w, c 2}}+\frac{\dot{Q}_{c 3}}{T_{w, c 3}}-\frac{\dot{Q}_{e 1}}{T_{w, e 1}}-\frac{\dot{Q}_{e 2}}{T_{w, e 2}}\right)+T_{H}\left(\frac{d S_{e 1}}{d t}+\frac{d S_{e 2}}{d t}+\frac{d S_{c 1}}{d t}+\frac{d S_{c 2}}{d t}+\frac{d S_{c 3}}{d t}\right) .
$$

\subsection{Optimal Control Design}

In Section 3 we derived an expression for the transient rate of exergy destruction in a VCS. This enables us to characterize the efficiency of the system dynamically from a second law perspective. Moreover, by utilizing techniques from the field of optimal control theory [8], we will use Eq. (42) as a minimization metric for operating the VCS at its maximum exergetic efficiency while meeting performance requirements.

In this section, an optimal controller is designed using total exergy destruction as the minimization metric. While there are many different optimal control algorithms, they all rely on the minimization of an objective or cost function to determine the optimal sequence of control actions for a particular system. However, many optimal control algorithms are applicable only to systems without input or output constraints. In the case of a second law, or exergetic optimization such as this one, it will be necessary to enforce constraints to ensure that thermodynamic laws are not violated. Moreover, for VCSs it is typical to have limits on actuators, etc. Therefore, we will use 
model predictive control (MPC), a receding-horizon optimal control framework that allows for constraints to be placed on input, output, and state variables [26].

\subsubsection{Prediction Model}

306 MPC uses a dynamic model to predict how the system will behave in response to a particular sequence of control decisions over a specified prediction horizon to influence control decisions at the current time step - this model is called the prediction model. The prediction horizon, $N_{p}$, is the number of discrete time steps over which the system behavior is predicted. It is defined as

$$
N_{p}=\frac{t_{\text {horizon }}}{\Delta t}
$$

where $\Delta t$ is the length of the discrete time step and $t_{\text {horizon }}$ is the length of time over which the algorithm predicts the

312 system behavior. The control horizon, $N_{u}$, is the number of discrete time steps for which control decisions are 313 optimized, where $N_{u} \leq N_{p}$. Figure 4 provides a visual interpretation of the MPC algorithm.

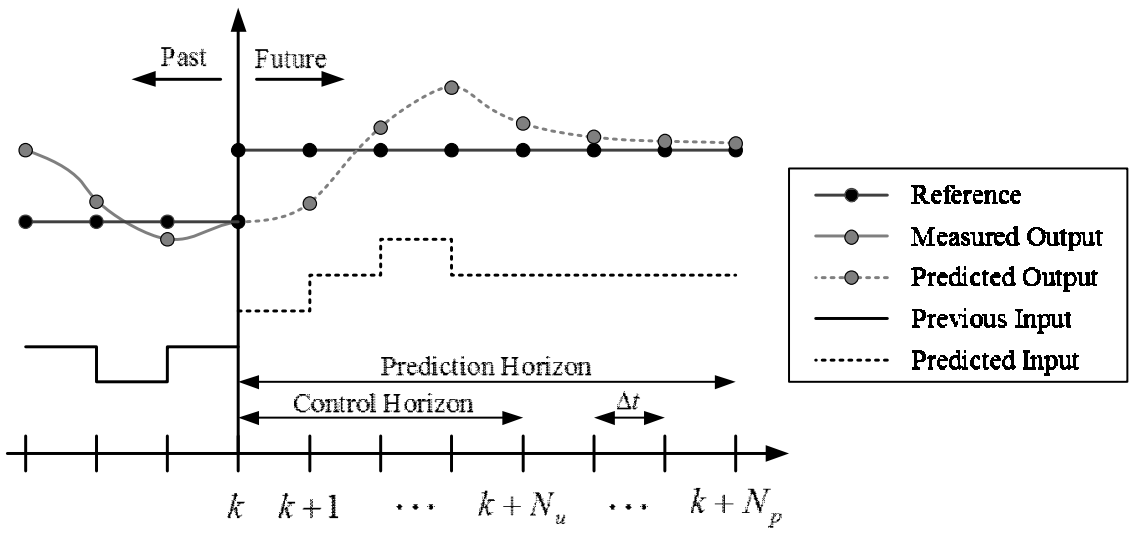

Figure 4: Schematic describing model predictive control.

316 To reduce computational complexity, a linear prediction model is preferred. Therefore, the nonlinear first-principles

317 VCS model used to derive Eq. (42) is linearized about an equilibrium point of the system; details of the model

318 linearization are provided in [31]. Moreover, since MPC is implemented in discrete time, the linearized model is 319 discretized at a sample time of $\Delta t$ and represented in a state space representation [32] as 


$$
\delta \mathbf{x}[k+1]=\mathbf{A} \delta \mathbf{x}[k]+\mathbf{B}_{1} \delta \mathbf{u}[k]+\mathbf{B}_{2} \delta \mathbf{d}[k]
$$

where $\delta \mathbf{x}, \delta \mathbf{u}$, and $\delta \mathbf{d}$ represent deviations from the equilibrium point, $\mathbf{A}$ is the state matrix, $\mathbf{B}_{1}$ is the control input matrix, and $\mathbf{B}_{2}$ is the disturbance input matrix. The state, input, and disturbance vectors for the VCS are described in Eqs. (45), (46), and (47), respectively. Note that instead of treating the evaporator and condenser fan speeds as decision variables, the air mass flow rates produced by each fan, $\dot{m}_{a, e}$ and $\dot{m}_{a, c}$, are used. The EEV aperture is described by $a_{v}$ and the compressor speed is described by $\omega_{k}$. Furthermore, to simplify the notation, the use of $\delta$ will be dropped since it is understood that we are discussing deviations about some nominal operating condition when referring to state, input, and disturbance variables.

$$
\begin{gathered}
\mathbf{x =}\left[\begin{array}{lllllllllllllll}
\zeta_{e 1} & P_{e} & h_{e 2} & T_{w, e 1} & T_{w, e 2} & \bar{\gamma}_{e} & \zeta_{c 1} & \zeta_{c 2} & P_{c} & h_{c 1} & h_{c 3} & T_{w, c 1} & T_{w, c 2} & T_{w, c 3} & \bar{\gamma}_{c}
\end{array}\right]^{T} \in \mathbb{R}^{15} \\
\mathbf{u}=\left[\begin{array}{lllll}
a_{v} & \omega_{k} & \dot{m}_{a, e} & \dot{m}_{a, c}
\end{array}\right]^{T} \in \mathbb{R}^{4} \\
\quad \mathbf{d}=\left[\begin{array}{lll}
T_{L} & T_{H}
\end{array}\right]^{T} \in \mathbb{R}^{2}
\end{gathered}
$$

Next we discuss a few modifications that are made to the prediction model. One can constrain the rate of change in control decisions over the control horizon by augmenting the system with additional states defined as $\mathbf{x}_{\mathbf{u}}[k]=\mathbf{u}[k-1]$ where $\mathbf{u}[k]=\mathbf{u}[k-1]+\Delta \mathbf{u}[k]$. Therefore, $\mathbf{x}_{\mathbf{u}}[k+1]=\mathbf{x}_{\mathbf{u}}[k]+\Delta \mathbf{u}[k]$ and the augmented statespace representation of the system is given by $\overline{\mathbf{x}}[k+1]=\overline{\mathbf{A}} \overline{\mathbf{x}}[k]+\overline{\mathbf{B}}_{1} \Delta \mathbf{u}[k]+\overline{\mathbf{B}}_{\mathbf{2}} \mathbf{d}[k]$, where

$$
\overline{\mathbf{x}}=\left(\begin{array}{c}
\mathbf{x} \\
\mathbf{x}_{\mathbf{u}}
\end{array}\right), \overline{\mathbf{A}}=\left(\begin{array}{cc}
\mathbf{A} & \mathbf{B}_{1} \\
\mathbf{0} & \mathbf{I}
\end{array}\right), \overline{\mathbf{B}}_{1}=\left(\begin{array}{c}
\mathbf{B}_{1} \\
\mathbf{I}
\end{array}\right), \overline{\mathbf{B}}_{2}=\left(\begin{array}{c}
\mathbf{B}_{2} \\
\mathbf{0}
\end{array}\right)
$$

For a numerical optimization, it is convenient to define the input vector in its lifted form, $\Delta \mathbf{U}=\left[\begin{array}{llll}\Delta \mathbf{u}[k] & \Delta \mathbf{u}[k+1] & \cdots & \Delta \mathbf{u}\left[k+n_{u}-1\right.\end{array}\right]^{T} . \quad$ Using the lifted input vector, $\Delta \mathbf{U}$, and the initial value of the state vector, $\overline{\mathbf{x}}[0]$, the evolution of all of the states can be quickly evaluated in the lifted vector $\overline{\mathbf{X}}$ using the lifted matrix equation $\overline{\mathbf{X}}=\mathbf{T} \overline{\mathbf{x}}[k]+\mathbf{S}_{\mathbf{1}} \Delta \mathbf{U}+\mathbf{S}_{\mathbf{2}} \mathbf{D}$ where $\overline{\mathbf{X}}=\left[\begin{array}{llll}\overline{\mathbf{x}}[k] & \overline{\mathbf{x}}[k+1] & \cdots & \overline{\mathbf{x}}\left[k+n_{p}-1\right]\end{array}\right]^{T} . \quad$ The expressions for $\mathbf{T}, \mathbf{S}_{\mathbf{1}}$, and $\mathbf{S}_{\mathbf{2}}$ are given by 


$$
\mathbf{T}=\left[\begin{array}{c}
\overline{\mathbf{A}} \\
\overline{\mathbf{A}}^{2} \\
\vdots \\
\overline{\mathbf{A}}^{n_{p}}
\end{array}\right], \mathbf{S}_{\mathbf{1}}=\left[\begin{array}{cccc}
\overline{\mathbf{B}}_{1} & 0 & \cdots & 0 \\
\overline{\mathbf{A}} \overline{\mathbf{B}}_{1} & \ddots & \ddots & \vdots \\
\vdots & \ddots & \overline{\mathbf{B}}_{\mathbf{1}} & 0 \\
\overline{\mathbf{A}}^{n_{u}-1} \overline{\mathbf{B}}_{1} & \cdots & \overline{\mathbf{A}} \overline{\mathbf{B}}_{1} & \overline{\mathbf{B}}_{\mathbf{1}} \\
\vdots & \vdots & \vdots & \vdots \\
\overline{\mathbf{A}}^{n_{p}-2} \overline{\mathbf{B}}_{1} & \ddots & \ddots & \overline{\mathbf{A}}^{n_{p}-n_{u}-1} \overline{\mathbf{B}}_{\mathbf{1}} \\
\overline{\mathbf{A}}^{n_{p}-1} \overline{\mathbf{B}}_{1} & \cdots & \overline{\mathbf{A}}^{n_{p}-n_{u}-1} \overline{\mathbf{B}}_{1} & \overline{\mathbf{A}}^{n_{p}-n_{u}} \overline{\mathbf{B}}_{\mathbf{1}}
\end{array}\right], \mathbf{S}_{2}=\left[\begin{array}{cccc}
\overline{\mathbf{B}}_{2} & 0 & \cdots & 0 \\
\overline{\mathbf{A}} \overline{\mathbf{B}}_{2} & \ddots & \ddots & \vdots \\
\vdots & \ddots & \overline{\mathbf{B}}_{\mathbf{2}} & 0 \\
\overline{\mathbf{A}}^{n_{u}-1} \overline{\mathbf{B}}_{2} & \cdots & \overline{\mathbf{A}} \overline{\mathbf{B}}_{2} & \overline{\mathbf{B}}_{2} \\
\vdots & \vdots & \vdots & \vdots \\
\overline{\mathbf{A}}^{n_{p}-2} \overline{\mathbf{B}}_{2} & \ddots & \ddots & \overline{\mathbf{A}}^{n_{p}-n_{u}-1} \overline{\mathbf{B}}_{2} \\
\overline{\mathbf{A}}^{n_{p}-1} \overline{\mathbf{B}}_{2} & \cdots & \overline{\mathbf{A}}^{n_{p}-n_{u}-1} \overline{\mathbf{B}}_{2} & \overline{\mathbf{A}}^{n_{p}-n_{u}} \overline{\mathbf{B}}_{2}
\end{array}\right] .
$$

The objective function, $J_{V C S, I I}$, that will be defined in the next section is a function of $\overline{\mathbf{X}}$.

\subsubsection{Objective Function}

For VCS operation we would like for the objective function to characterize both the efficiency and performance of the system. Since the MPC considers a receding finite time-horizon, the total exergy destroyed over the prediction horizon will be minimized. The performance objective is defined as the 2-norm of the difference between the desired cooling capacity (as specified by the user) and the cooling capacity achieved by the VCS over the prediction horizon. A weighting parameter, $\lambda$, is used to emphasize the importance of one objective over the other. Finally, as mentioned earlier, the MPC will be implemented in discrete time; therefore, numerical integration will be used to approximate the total exergy destroyed over the prediction horizon.

The complete second law objective function, $J_{V C S, I I}$, is expressed as

$$
J_{V C S, I I}=\underbrace{\left(\left\|C_{\text {des }}-C_{a c h}\right\|_{2}\right)}_{\begin{array}{c}
\text { Performance } \\
\text { Objective }
\end{array}}+\lambda \cdot \underbrace{\left(\sum_{k=1}^{N_{p}} \dot{X}_{\text {dest,VCS}}[k]\right) \Delta t}_{\begin{array}{l}
\text { Efficiency } \\
\text { Objective }
\end{array}} .
$$

Substituting Eq. (3) into Eq. (50) and simplifying yields

$$
\begin{aligned}
J_{V C S, I I} & =\left\|C_{d e s}-C_{a c h}\right\|_{2}+\lambda \cdot T_{H} \cdot \Delta t\left(\sum_{k=1}^{N_{p}} \dot{S}_{g e n, V C S}[k]\right) \\
& =\left\|C_{d e s}-C_{a c h}\right\|_{2}+\bar{\lambda}\left(\sum_{k=1}^{N_{p}} \dot{S}_{g e n, V C S}[k]\right)
\end{aligned}
$$

where the reference (dead state) temperature, $T_{H}$, and the discrete sample time, $\Delta t$, are absorbed into the weighting parameter $\bar{\lambda}$. The theoretical minimum of the objective function shown in Eq. (51) is zero. 
Remark 1. The choice of the correct reference temperature becomes less critical in this formulation where $T_{H}$ is absorbed into the weighting parameter, $\bar{\lambda}$. In fact, $T_{H}$ plays little role in the tradeoff between the performance and

362 efficiency objectives from the perspective of the optimization algorithm assuming the user heuristically tunes $\bar{\lambda}$ for 363 the desired tracking performance of the controller. This is a feature for many thermal systems as it can be difficult 364 to define the correct reference state for certain applications and problems [33] [34].

Remark 2. In Eq. (50), $\dot{X}_{\text {dest }}$ represents the instantaneous rate of exergy destruction and will take on a constant value at steady-state, unlike a time differential which will become zero at steady-state. Therefore, the expression $\dot{X}_{\text {dest, }, C S}[k]$ refers to the rate of exergy destruction in the VCS at some time instant $k$. Similarly, in Eq. (51), $\dot{S}_{g e n}$ is the instantaneous rate of entropy generation, and $\dot{S}_{g e n, V C S}[k]$ refers to the rate of entropy generation in the VCS at some time instant $k$.

The desired cooling capacity, $C_{d e s} \in \mathbb{R}^{N_{p}}$, is specified by the user in the optimization problem, and $C_{a c h} \in \mathbb{R}^{N_{p}}$ is calculated using the expression

$$
C_{a c h}=\dot{Q}_{e}=(U A)_{e 1}\left(T_{w, e 1}-T_{r, e 1}\right)+(U A)_{e 2}\left(T_{w, e 2}-T_{r, e 2}\right)
$$

The efficiency objective can be expanded as

$$
\begin{aligned}
\sum_{k=1}^{N_{p}} \dot{S}_{g e n, V C S}[k]= & \sum_{k=1}^{N_{p}}\left(\frac{\dot{Q}_{c 1}[k]}{T_{w, c 1}[k]}+\frac{\dot{Q}_{c 2}[k]}{T_{w, c 2}[k]}+\frac{\dot{Q}_{c 3}[k]}{T_{w, c 3}[k]}-\frac{\dot{Q}_{e 1}[k]}{T_{w, e 1}[k]}-\frac{\dot{Q}_{e 2}[k]}{T_{w, e 2}[k]}\right) \\
& +\sum_{k=1}^{N_{p}}\left(g_{e 1}[k]+g_{e 2}[k]+g_{c 1}[k]+g_{c 2}[k]+g_{c 3}[k]\right)
\end{aligned}
$$

where

$$
\dot{Q}_{e i}[k]=(U A)_{e i}[k] \cdot\left(T_{w, e i}[k]-T_{r, e i}[k]\right), i \in\{1,2\},
$$

$$
\dot{Q}_{c i}[k]=(U A)_{c i}[k] \cdot\left(T_{r, c i}[k]-T_{w, c i}[k]\right), i \in\{1,2,3\},
$$




$$
\begin{aligned}
g_{e 1}[k] & =s_{e 1}[k]\left(\dot{m}_{r, v}[k]-\dot{m}_{e 12}[k]+L_{R, e} A_{C R, e} \rho_{e 1}[k]\left(\frac{\zeta_{e 1}[k]-\zeta_{e 1}[k-1]}{\Delta t}\right)\right) \\
& +L_{R, e} A_{C R, e} \rho_{e 1}[k] \zeta_{e 1}[k]\left(\frac{\left(\partial s_{e 1}\right)}{\partial \bar{\gamma}_{e}}[k]\left(\frac{\bar{\gamma}_{e}[k]-\bar{\gamma}_{e}[k-1]}{\Delta t}\right)+\frac{\left(\partial s_{e 1}\right)}{\partial P_{e}}[k]\left(\frac{P_{e}[k]-P_{e}[k-1]}{\Delta t}\right)\right),
\end{aligned}
$$

$$
g_{e 2}[k]=s_{e 2}[k]\left(\dot{m}_{e 12}[k]-\dot{m}_{r, k}[k]+L_{R, e} A_{C R, e} \rho_{g, e}[k]\left(\frac{\zeta_{e 1}[k]-\zeta_{e 1}[k-1]}{\Delta t}\right)\right)
$$

$$
+L_{R, e} A_{C R, e} \rho_{e 2}[k] \zeta_{e 2}[k]\left(\frac{\left(\partial s_{e 2}\right)}{\partial h_{e 2}}[k]\left(\frac{h_{e 2}[k]-h_{e 2}[k-1]}{\Delta t}\right)+\frac{\left(\partial s_{e 2}\right)}{\partial P_{e}}[k]\left(\frac{P_{e}[k]-P_{e}[k-1]}{\Delta t}\right)\right),
$$

$$
\begin{aligned}
g_{c 1}[k] & =s_{c 1}[k]\left(\dot{m}_{r, k}[k]-\dot{m}_{c 12}[k]+L_{R, c} A_{C R, c} \rho_{g, c}[k]\left(\frac{\zeta_{c 1}[k]-\zeta_{c 1}[k-1]}{\Delta t}\right)\right) \\
& +L_{R, c} A_{C R, c} \rho_{c 1}[k] \zeta_{c 1}[k]\left(\frac{\left(\partial s_{c 1}\right)}{\partial h_{c 1}}[k]\left(\frac{h_{c 1}[k]-h_{c 1}[k-1]}{\Delta t}\right)+\frac{\left(\partial s_{c 1}\right)}{\partial P_{c}}[k]\left(\frac{P_{c}[k]-P_{c}[k-1]}{\Delta t}\right)\right),
\end{aligned}
$$

$$
\begin{aligned}
g_{c 2}[k] & =s_{c 2}[k]\left(\dot{m}_{c 12}[k]-\dot{m}_{c 23}[k]+L_{R, c} A_{C R, c} \rho_{l, c}[k]\left(\frac{\zeta_{c 1}[k]-\zeta_{c 1}[k-1]}{\Delta t}+\frac{\zeta_{c 2}[k]-\zeta_{c 2}[k-1]}{\Delta t}\right)\right) \\
& -S_{c 2}[k]\left(L_{R, c} A_{C R, c} \rho_{g, c}[k]\left(\frac{\zeta_{c 1}[k]-\zeta_{c 1}[k-1]}{\Delta t}\right)\right) \\
& +L_{R, c} A_{C R, c} \rho_{c 2}[k] \zeta_{c 2}[k]\left(\frac{\left(\partial s_{c 2}\right)}{\partial \bar{\gamma}_{c}}[k]\left(\frac{\bar{\gamma}_{c}[k]-\bar{\gamma}_{c}[k-1]}{\Delta t}\right)+\frac{\left(\partial s_{c 2}\right)}{\partial P_{c}}[k]\left(\frac{P_{c}[k]-P_{c}[k-1]}{\Delta t}\right)\right),
\end{aligned}
$$

and

$$
\begin{aligned}
g_{c 3}[k] & =s_{c 3}[k]\left(\dot{m}_{c 23}[k]-\dot{m}_{r, v}[k]-L_{R, c} A_{C R, c} \rho_{l, c}[k]\left(\frac{\zeta_{c 1}[k]-\zeta_{c 1}[k-1]}{\Delta t}+\frac{\zeta_{c 2}[k]-\zeta_{c 2}[k-1]}{\Delta t}\right)\right) \\
& +L_{R, c} A_{C R, c} \rho_{c 3}[k] \zeta_{c 3}[k]\left(\frac{\left(\partial s_{c 3}\right)}{\partial h_{c 3}}[k]\left(\frac{h_{c 3}[k]-h_{c 3}[k-1]}{\Delta t}\right)+\frac{\left(\partial s_{c 3}\right)}{\partial P_{c}}[k]\left(\frac{P_{c}[k]-P_{c}[k-1]}{\Delta t}\right)\right) .
\end{aligned}
$$

For clarity of notation, the rate of change of entropy in each evaporator control volume is denoted by the expression $g_{e i}$ where $i=\{1,2\}$. Similarly, in each condenser control volume, the rate of change of entropy is denoted by the expression $g_{c i}$ where $i=\{1,2,3\}$. It should also be noted that $J_{V C S, I I}$ is not only a function of the states of the dynamical system representation of the VCS but also a function of variables such as $T_{r, c 1}$ and $\partial s_{e 2} / \partial h$ which are nonlinear functions of the states. These variables are typically evaluated using data-based refrigerant look-up tables [30]. Therefore, while the dynamic prediction model itself is linear, the objective function is nonlinear. 


\subsubsection{Constraints}

397 In the MPC framework, upper and lower bound constraints can easily be placed on the values of the control decisions at each time instant. The constraint values are detailed in Section 5.1 for the specific VCS considered in the case study. Additionally, upper and/or lower bound constraints can be enforced on specific state variables in the dynamical system. For VCS operation, we typically seek nonzero superheat in the evaporator and nonzero subcooling in the condenser [12]. Therefore, the constraints defined in Eq. (61) and Eq. (62) ensure that the normalized lengths of the superheated fluid region in the evaporator and subcooled region in the condenser, respectively, are maintained at some minimum fraction of the total tube length in each heat exchanger.

$$
\begin{gathered}
\zeta_{e 1}[k] \leq 0.95 \forall k \\
\zeta_{c 1}[k]+\zeta_{c 2}[k] \leq 0.95 \forall k
\end{gathered}
$$

Finally, the following nonlinear constraints are introduced to satisfy the second law of thermodynamics:

$$
\dot{W}_{V C S}[k]-\dot{X}_{V C S}[k] \geq 0 \forall k
$$

where $\dot{W}_{V C S}=\dot{W}_{k}$ is equal to the power (energy consumption rate) of the compressor and

$$
\begin{aligned}
& \dot{X}_{\text {dest }, k}[k] \geq 0 \forall k, \\
& \dot{X}_{\text {dest, },}[k] \geq 0 \forall k, \\
& \dot{X}_{\text {dest }, e}[k] \geq 0 \forall k, \\
& \dot{X}_{\text {dest }, c}[k] \geq 0 \forall k .
\end{aligned}
$$

Equation (63) ensures that the reversible power is always nonnegative, and the inequalities shown in Eq. (64) ensure that the exergy destruction rate for each individual component of the VCS is always nonnegative.

\section{RESULTS AND DISCUSSION}

In this section, a case study is presented in which the exergy-based (second law) model predictive controller (MPC) is implemented on a simulated VCS; the details of the case study and the closed-loop results will be presented in Section 4.1. In Section 4.2, an energy-based (first law) MPC will be designed and implemented on the same VCS, and the simulation results will be compared against the results presented in Section 5.1. The tradeoffs between the first law and second law optimal controllers will be discussed, specifically in the context of transient VCS operation. 
422 The exergy-based (second law) MPC was implemented in simulation on a linearized VCS model. The nonlinear 423 VCS model has been validated against an experimental system at the University of Illinois at Urbana-Champaign 424 described in [35], and the linearization procedure is described in [31]. The model describes a 1kW capacity vapor compression system operating with R134a refrigerant and consists of a semi-hermetic reciprocating compressor, electronic expansion valve, condenser, and evaporator, with variable-speed heat exchanger fans. The function fmincon in the MATLAB Optimization Toolbox was used with a sequential quadratic programming algorithm to implement the model predictive controller. The desired cooling capacity, $C_{d e s}$, is shown in Figure 5. This reference trajectory was chosen to elicit the transient behavior that results from high frequency loading in cooling applications such as refrigerated food transport.

431

432

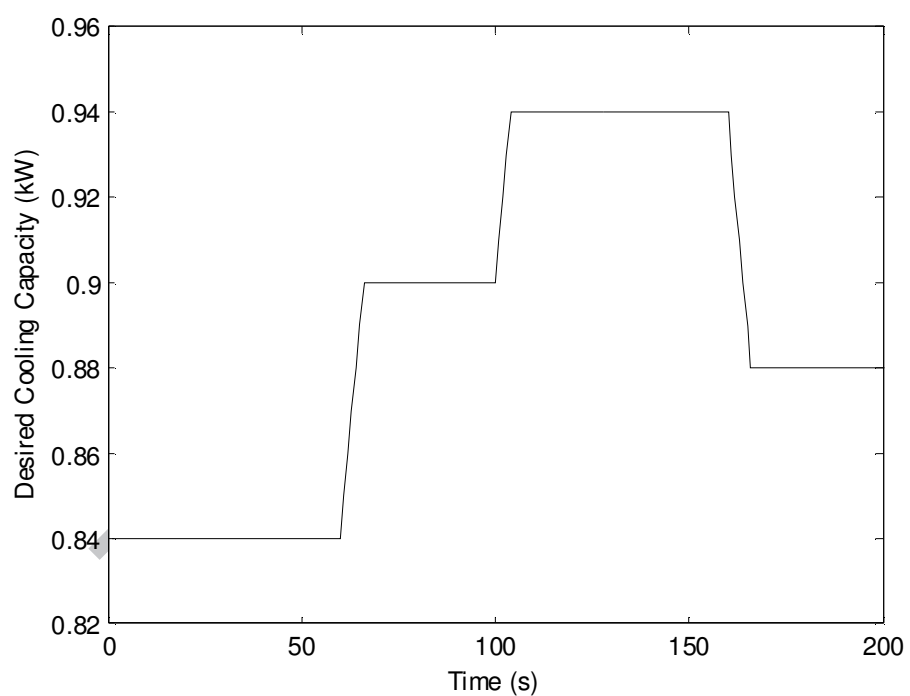

Figure 5: Cooling capacity reference trajectory.

The length of the prediction horizon and the control horizon were chosen as $N_{p}=N_{u}=15$, with a sample time, $\Delta t$, of 1 second. For this case study, it was assumed that the reference trajectory was known a priori. The weighting factor $\lambda$ was chosen heuristically as $8 \times 10^{-3}$ to sufficiently weight the performance objective and achieve reasonable reference tracking performance. The constant disturbances (Eq. (47)) were specified as $T_{L}=18^{\circ} \mathrm{C}$ and $T_{H}=26^{\circ} \mathrm{C}$ where $T_{L}$ is the temperature of the low-temperature reservoir interacting with the evaporator and $T_{H}$ is the temperature of the ambient environment. Finally, the upper and lower bound constraints on the decision variables 

and condenser air mass flow rates, respectively.

Table 1: Upper and Lower Bound Constraints on Decision Variables

\begin{tabular}{cccc}
\hline \hline $\begin{array}{c}\text { Decision } \\
\text { Variable }\end{array}$ & Units & Lower Bound & Upper Bound \\
\hline$a_{v}$ & $\%$ open & 8 & 11 \\
$\omega_{k}$ & $\mathrm{rpm}$ & 900 & 1100 \\
$\dot{m}_{a, e}$ & $\mathrm{~kg} / \mathrm{s}$ & 0.1 & 0.3 \\
$\dot{m}_{a, c}$ & $\mathrm{~kg} / \mathrm{s}$ & 0.3 & 0.65 \\
\hline \hline
\end{tabular}

443

444 The tracking performance of the second law MPC is shown in Figure 6. The control input signals are shown in 445 Figure 7, and the exergy destruction rate and the exergetic efficiency are plotted in Figure 8.

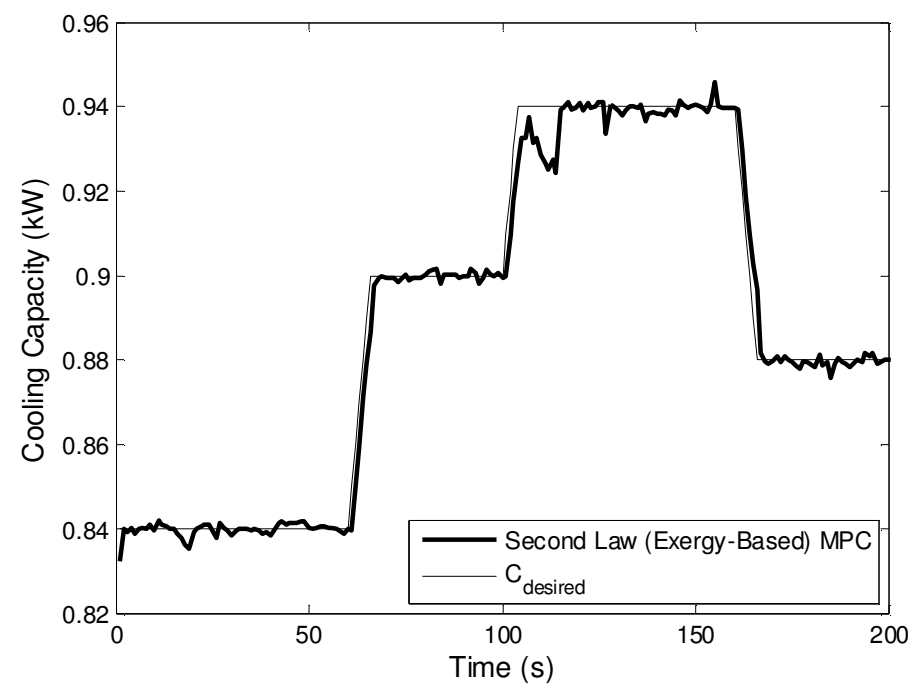



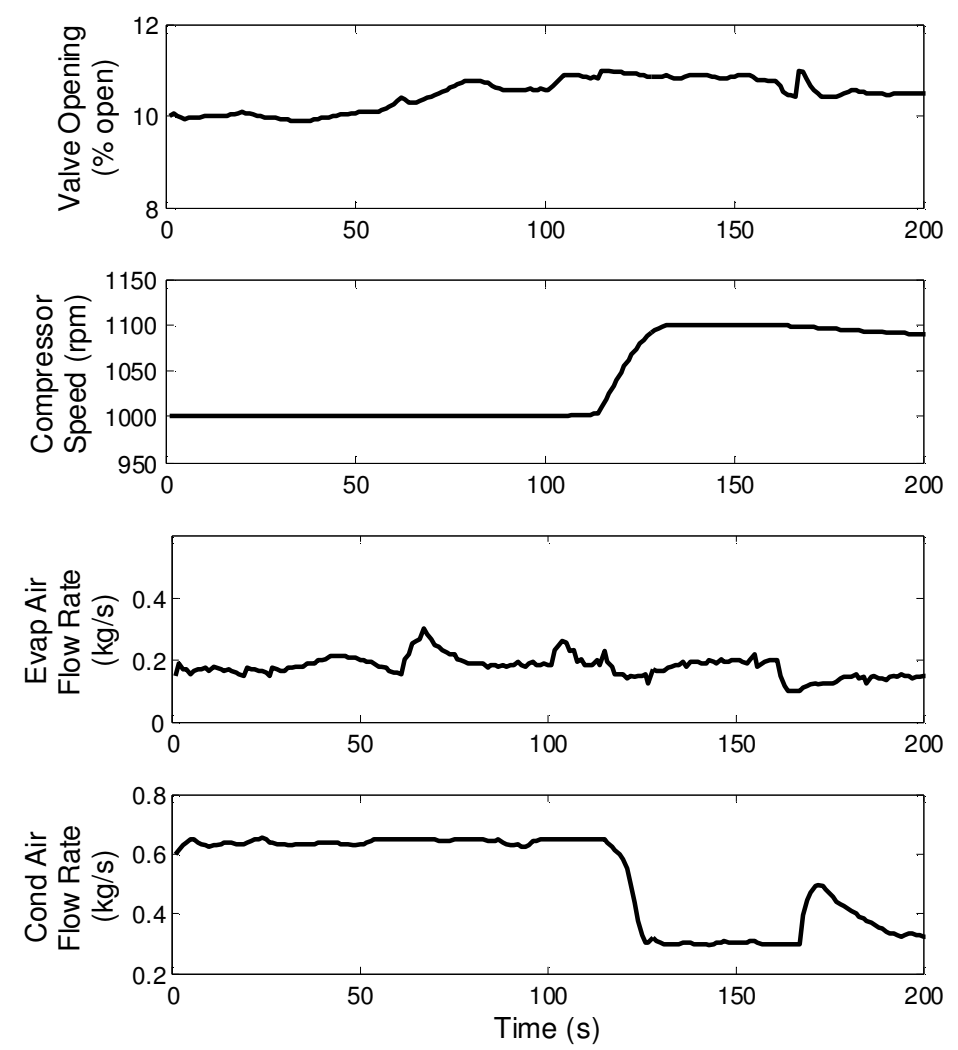

Figure 7: Second law (exergy-based) MPC - control input signals.
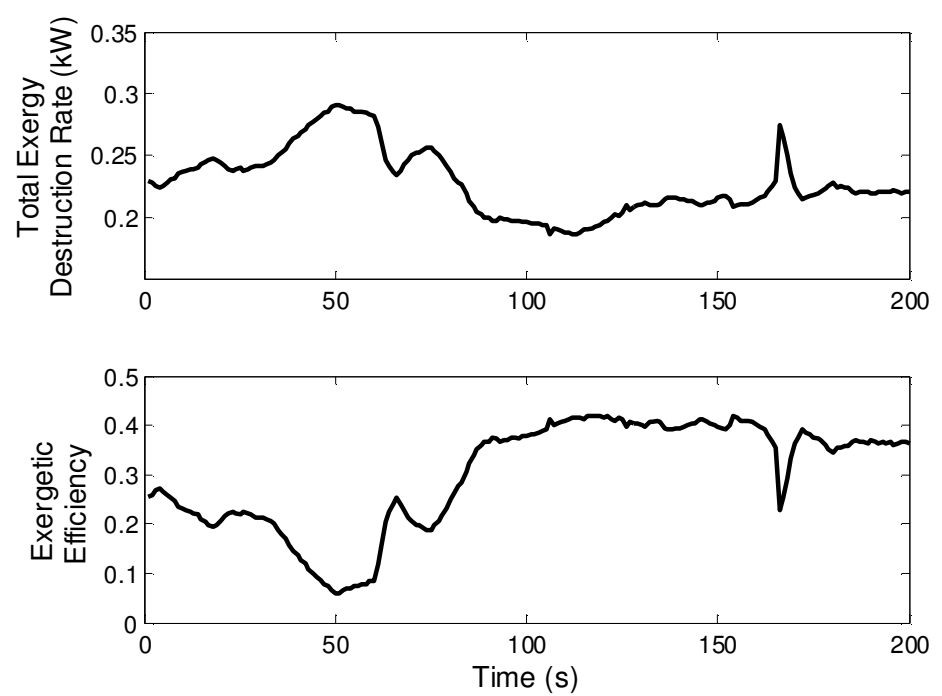


\subsubsection{Reversible Work Analysis}

Recall Eq. (5) written here in non-rate form:

$$
X_{\text {dest }}=W-W_{r e v} \text {. }
$$

Using Eq. (65), the reversible work during a finite time-horizon assuming transient operation of the VCS is

$$
\begin{aligned}
W_{r e v}= & W-X_{\text {dest }} \\
= & \sum_{k=1}^{N_{p}}\left(\dot{m}_{r, k}[k]\left(h_{k, r o}[k]-h_{k, r i}[k]\right)\right)-T_{H} \sum_{k=1}^{n_{p}}\left(\frac{\dot{Q}_{c 1}[k]}{T_{w, c 1}[k]}+\frac{\dot{Q}_{c 2}[k]}{T_{w, c 2}[k]}+\frac{\dot{Q}_{c 3}[k]}{T_{w, c 3}[k]}-\frac{\dot{Q}_{e 1}[k]}{T_{w, e 1}[k]}-\frac{\dot{Q}_{e 2}[k]}{T_{w, e 2}[k]}\right) \\
& -T_{H} \sum_{k=1}^{N_{p}}\left(g_{e 1}[k]+g_{e 2}[k]+g_{c 1}[k]+g_{c 2}[k]+g_{c 3}[k]\right)
\end{aligned}
$$

which will not be constant with respect to the decision variables (the control input sequence $\Delta \mathbf{U}$ ) each time the MPC problem is solved. Therefore, we expect that an MPC designed to minimize a first law objective will produce different results than were presented in the previous section. We will now design another model predictive controller where the objective function, $J_{V C S, I}$, is formulated to minimize the total energy consumed over the prediction horizon:

$$
\begin{aligned}
J_{V C S, I} & =\left(\left\|C_{d e s}-C_{a c h}\right\|_{2}\right)+\lambda \cdot \Delta t\left(\sum_{k=1}^{N_{p}} \dot{W}_{k}[k]\right) \\
& =\left(\left\|C_{d e s}-C_{a c h}\right\|_{2}\right)+\lambda \cdot \Delta t\left(\sum_{k=1}^{N_{p}} \dot{m}_{k}[k]\left(h_{k, r o}[k]-h_{k, r i}[k]\right)\right),
\end{aligned}
$$

where $\dot{W}_{k}$ is the instantaneous power consumption (i.e. energy consumption rate) in the VCS. The first law MPC is designed with the same constraints, weighting factor $\lambda$, and constant disturbances that were specified in the second law MPC. The closed-loop results of the two controllers will be compared in the following section.

\subsubsection{Comparison of Closed-Loop Simulation Results}

First the tracking of the desired cooling capacity by each optimal controller is compared in Figure 9. As expected, both controllers produce very similar results. 


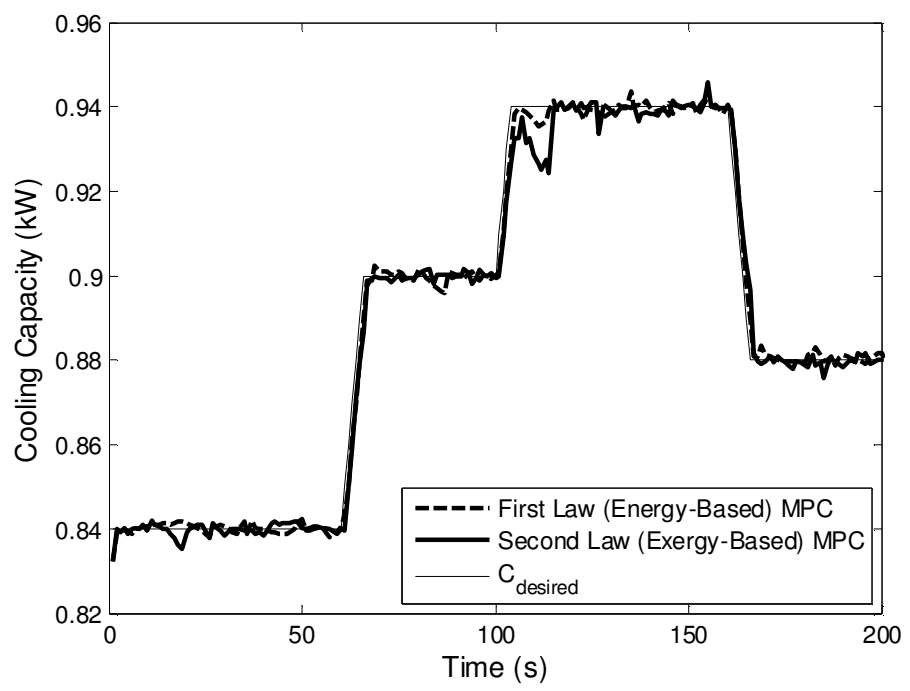

Figure 9: Comparison of cooling capacity tracking performance using first and second law MPC.

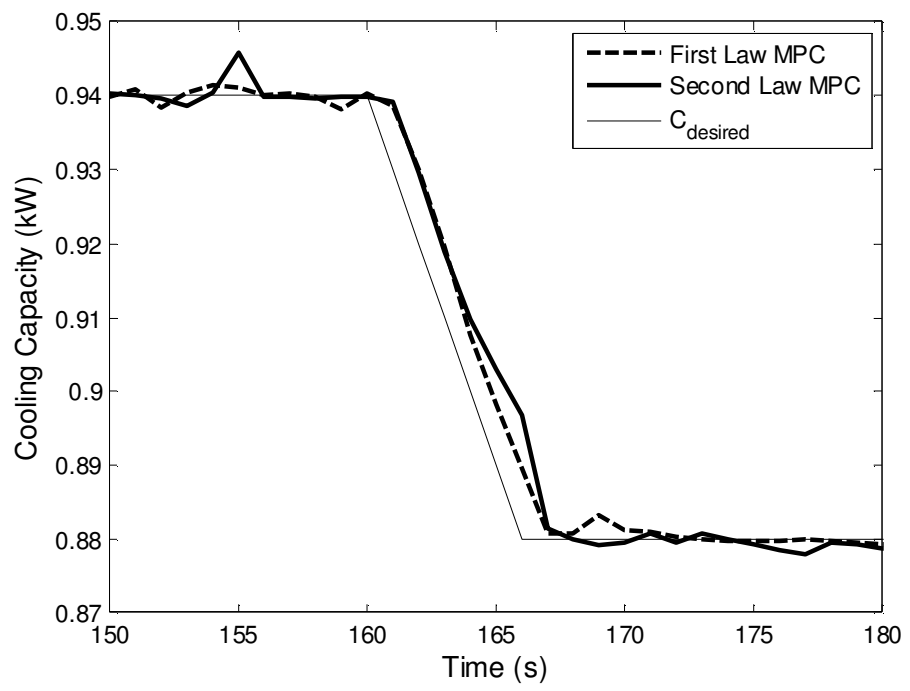

Figure 10: Closer view of Figure 9. 

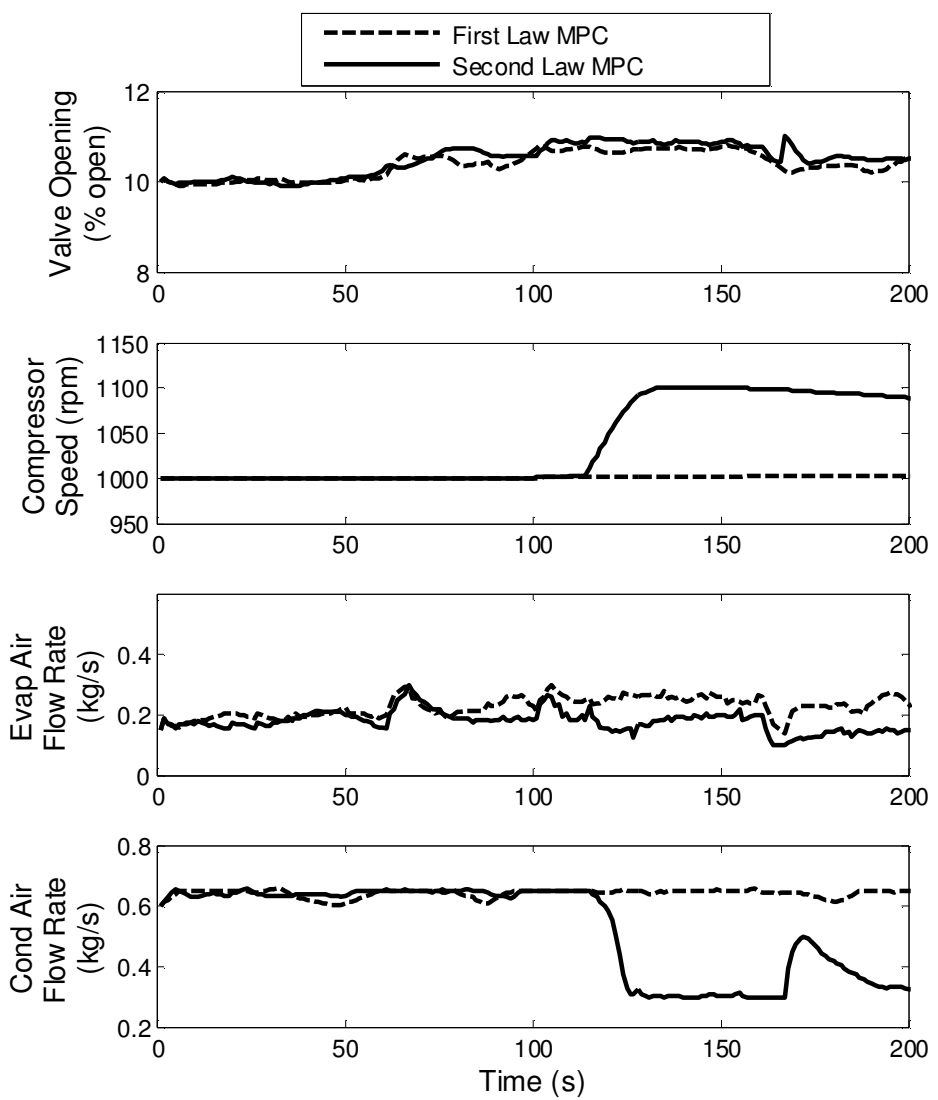

Figure 11: Comparison of control input signals using each of the two controllers.

482 The control input signals associated with each controller are compared in Figure 11. The primary difference is seen 483 in the input signals for the compressor and the condenser air mass flow rate. In particular, the first law MPC 484 resulted in the condenser air mass flow rate at its maximum allowable value $(0.65 \mathrm{~kg} / \mathrm{s})$ for most of the 200 -second time horizon whereas the second law MPC dropped the condenser air mass flow rate to its lower bound $(0.3 \mathrm{~kg} / \mathrm{s})$ for much of the latter part of the simulation. During this time the first law MPC kept the compressor speed constant 487 where the second law MPC increased the value to $1100 \mathrm{rpm}$.

Remark 3. To better characterize and understand transient exergy destruction in a VCS, only the refrigerant side dynamics of the VCS were considered in the derivation. Therefore the power consumption of the heat exchanger 491 fans is not considered in either objective function, implying that there is no penalty, from a first law perspective, of 492 choosing high evaporator and condenser air mass flow rates. This can explain why the first law MPC resulted in 493 higher evaporator and condenser air mass flow rates than the second law MPC. However, operating the VCS with 
494 high air mass flow rates has consequences with regards to the exergy destruction in the evaporator and condenser 495 which are penalized by the second law MPC. This will be highlighted later in Figure 13.

497 The exergy destruction rate, energy consumption rate, and reversible power resulting from each of the controllers 498 are shown in Figure 12. First, it is important to highlight that the reversible power is not equivalent between the two 499 simulations, verifying the statement made earlier regarding the expected difference in the two controllers. This is 500 particularly important because transient exergy analyses are not typically applied to VCSs nor is exergy destruction 501 minimization typically conducted using a transient exergy destruction rate. These results show that when 502 considering transient operation of a VCS, an exergy-based optimal controller has the potential to make different 503 decisions about how to operate the system than a conventional energy-based controller will, to meet the same 504 performance demand. Also as expected, the second law MPC destroyed less exergy over the 200-second simulation 505 whereas the first law MPC consumed less energy during the same simulation. The total exergy destroyed and total 506 energy consumed using each controller is compared in Table 2. Note that the percent differences were calculated 507 relative to the performance of the second law MPC. 

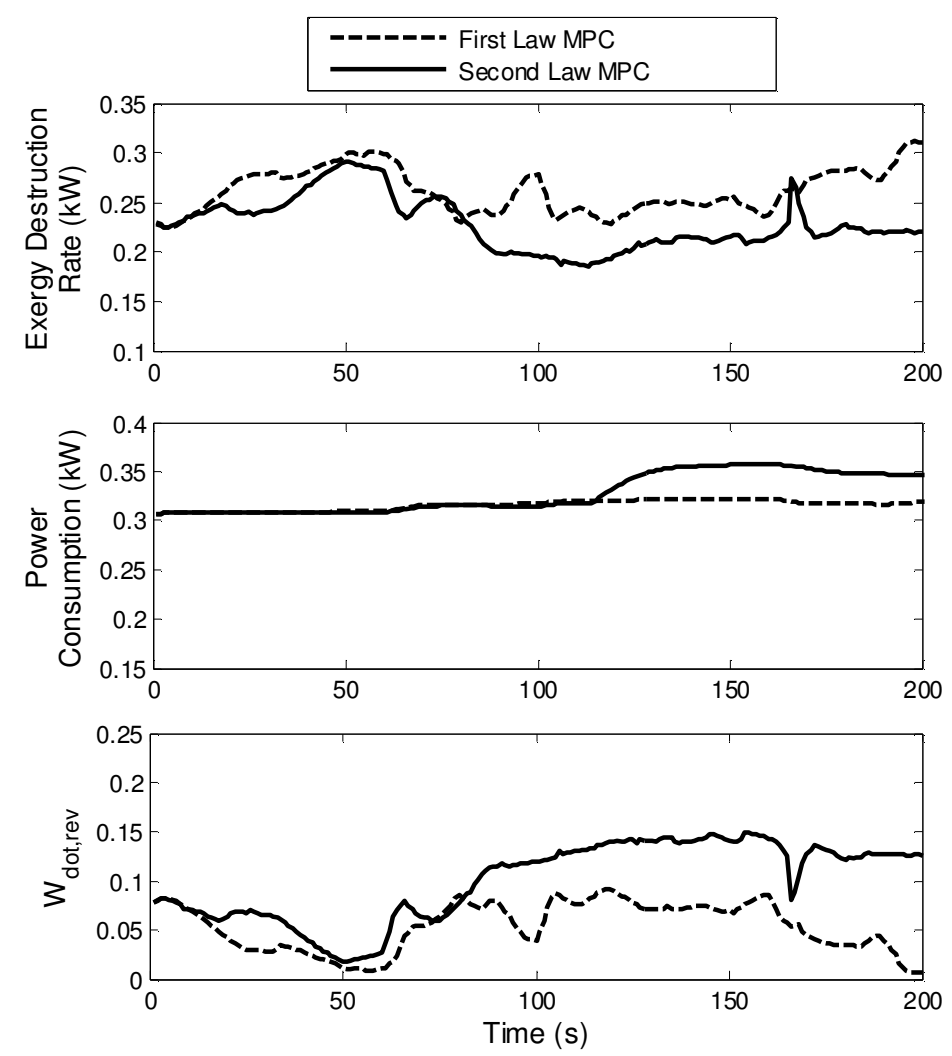

Figure 12: Comparison of exergy destruction rate, energy consumption rate, and reversible power using each of the two controllers. The same range is used for each plot.

513 Table 2: Total Exergy Destruction and Energy Consumption Using Each Controller

\begin{tabular}{cccc}
\hline \hline & Second Law MPC & First Law MPC & $\begin{array}{c}\text { Percent } \\
\text { Difference (\%) }\end{array}$ \\
\hline Total Exergy Destroyed (kJ) & 45.6 & 52.4 & -14.9 \\
Total Energy Consumed (kJ) & 65.6 & 63.1 & 3.76 \\
\hline \hline
\end{tabular}

Although the second law MPC consumes 3.76\% more energy than the first law MPC, it destroys almost 15\% less exergy. Therefore, the tradeoff between energy consumption and exergy destruction is not necessarily 1:1. To

517 analyze this more closely, the exergy destruction rate for each individual VCS component is compared in Figure 13.

518 The total exergy destroyed and energy consumed during the complete simulation in each component using both 519 controllers is shown in Table 3. 
Table 3: Total Exergy Destruction Evaluated by Component Using Each Controller

\begin{tabular}{cccc}
\hline \hline $\begin{array}{c}\text { Total Exergy Destruction } \\
\text { by Component }\end{array}$ & Second Law MPC & First Law MPC & $\begin{array}{c}\text { Percent } \\
\text { Difference (\%) }\end{array}$ \\
\hline EEV & $3.12 \mathrm{~kJ}$ & $2.83 \mathrm{~kJ}$ & 9.45 \\
Compressor & $33.7 \mathrm{~kJ}$ & $32.1 \mathrm{~kJ}$ & 4.86 \\
Evaporator & $2.06 \mathrm{~kJ}$ & $3.81 \mathrm{~kJ}$ & -85.4 \\
Condenser & $6.73 \mathrm{~kJ}$ & $13.7 \mathrm{~kJ}$ & -103 \\
\hline \hline
\end{tabular}

524

525

526

527

528

529

530

531

532

533

534

535

536

537

Surprisingly, the first law MPC destroyed less exergy in the EEV and compressor. However, in the evaporator and condenser, the first law MPC destroyed $103 \%$ and $85.4 \%$ more exergy, respectively, than was destroyed using the second law MPC.

Remark 4. It has long been cited that the greatest exergy destruction site in a VCS is the compressor [1]. This is still the case as shown in Table 3. However, these results show that it is possible for exergy to be destroyed on the same order of magnitude in other components, in this case the condenser, when all four control inputs are being modulated. As variable-speed fans become more common in commercial VCSs, the effect of air mass flow rate on the overall efficiency of the system can be quite significant, particularly during transient operation. What is more, the irreversibilities being characterized here are not of the fans themselves (which is omitted from this analysis) but from heat transfer and mass transfer occurring inside the evaporator and condenser. These irreversibilities are inherently not taken into account in an energy-based controller, which would only be able to account for losses (e.g. due to pressure drop) in the fans which drive air across the heat exchangers. 

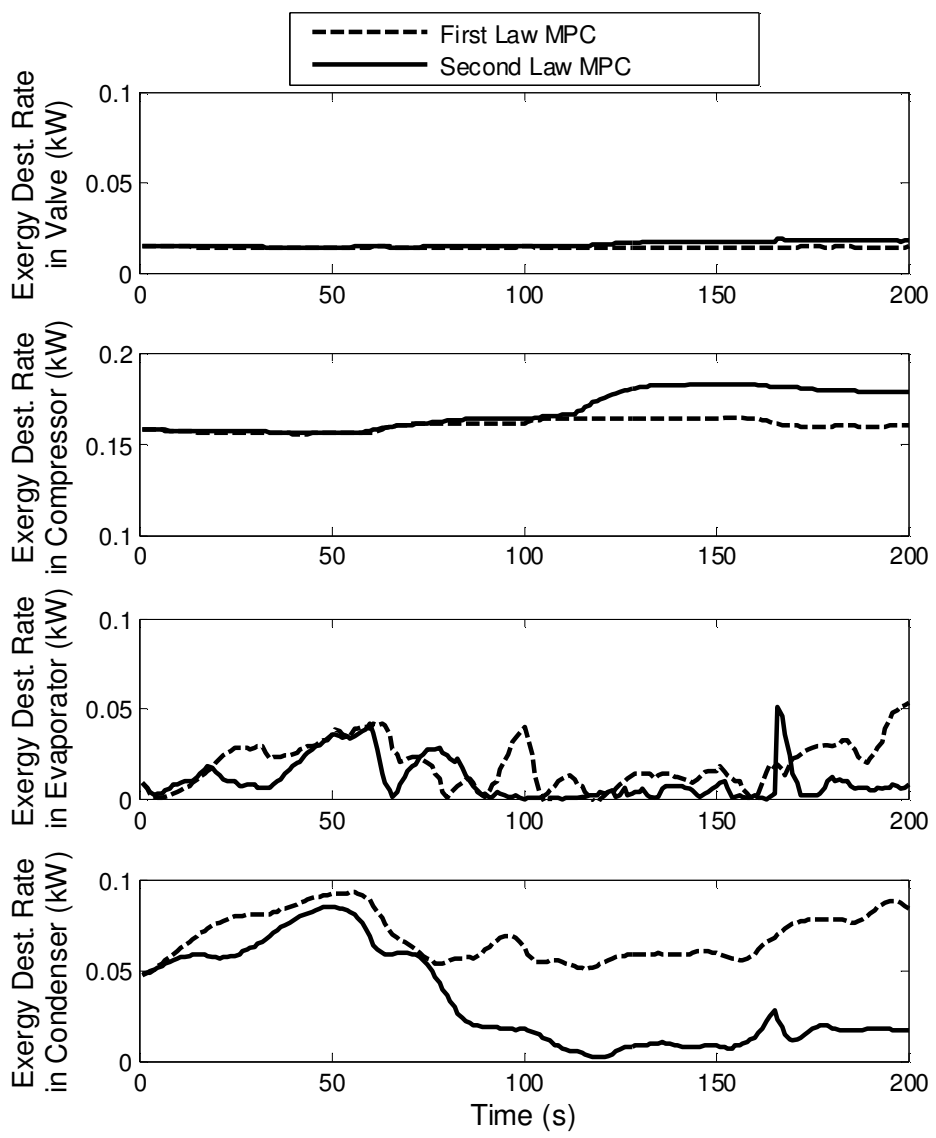

Figure 13: Exergy destruction rate comparison by VCS component for each of the two controllers. The same range is used for each plot.

541 Finally, we evaluate two efficiency metrics for VCSs - the COP and the exergetic efficiency - for transient operation as shown in Eq. (68) and Eq. (69) respectively.

$$
\begin{gathered}
\mathrm{COP}[k]=\frac{\dot{Q}_{e}[k]}{\dot{W}_{k}[k]} \\
\eta_{I I}[k]=1-\frac{\dot{X}_{d e s t, V C C}[k]}{W_{k}[k]}
\end{gathered}
$$

COP is a measure of the rate at which cooling is achieved by the VCS, relative to the rate at which work is done on the system. By virtue of how VCSs work, the COP is generally greater than one, with a higher COP indicating

547 greater efficiency. A major downside of COP as a metric, however, is that it is inherently not normalized, so it 548 cannot be used to characterize how well a system is performing relative to a baseline measure of performance. 549 Alternatively, the exergetic efficiency measures the rate at which exergy is destroyed relative to the rate at which 
exergy is supplied to the VCS. This metric is defined between 0 and 1 and tells us how effectively the exergy supplied to this system, in this case work done on the compressor, is used in the VCS in an absolute sense.

Figure 14 shows that the first law MPC operates the system at a higher COP but with a lower exergetic efficiency. On average, the COP achieved by the second law MPC is 3.95\% lower than that achieved by the first law MPC. On the other hand, on average, the exergetic efficiency achieved by the second law MPC is $41.4 \%$ greater than that achieved by the first law MPC. These results indicate that although the second law MPC would operate the system in such a way as to consume slightly more energy, that energy is being used by the system more effectively. To be more precise, this means operating the system with fewer irreversibilities, such as friction in refrigerant flow and losses in heat transfer across finite temperature differences. In the case of VCSs with thermal storage systems, this may allow one to achieve cooling more efficiently by producing and storing excess cooling at particular times, as opposed to simply minimizing energy consumption at all times. Operating the system using an exergy-based optimal controller can also have implications on the wear of the physical components themselves, a longer term

564 in this case study.

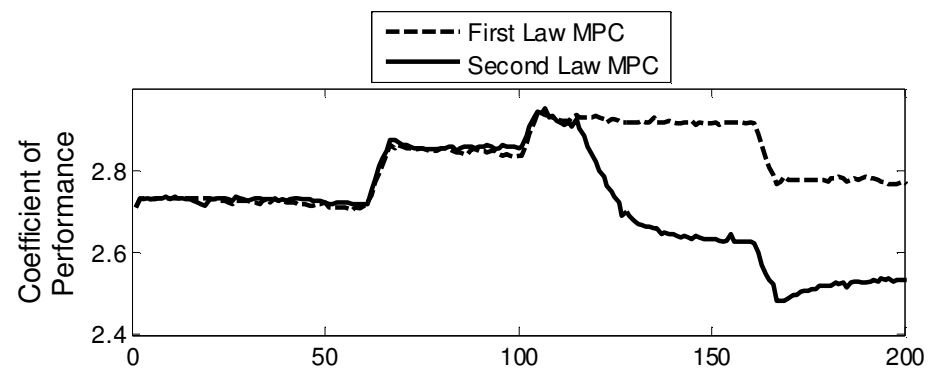


571 In this paper we derived an expression for the transient rate of exergy destruction for the refrigerant-side dynamics

572 of a VCS that in turn was used to design and implement an exergy-based model predictive controller for closed-loop

573 operation of the VCS. Simulation results showed that during transient operation of the VCS, the second law

574 (exergy-based) MPC specifically accounted for irreversibilities in each component of the system whereas a

575 comparable first law MPC did not. The distribution of irreversibilities across the heat exchangers, in particular,

576 varied significantly between the second law and first law model predictive controllers. Moreover, the distribution of

577 exergy destruction across the components of the VCS changed as a function of the control inputs, demonstrating not

578 only the importance of considering the dynamic exergy destruction rate but also of optimal control of VCSs with full

579 actuation. The results presented here consider a canonical VCS and therefore, future work will focus on applying

580 the tools developed in this paper to an experimental VCS to validate the use of exergy-based optimal control.

NOMENCLATURE

$\begin{array}{cl}A & \text { area } \\ a & \text { aperture } \\ C & \text { cooling capacity } \\ E & \text { energy } \\ J & \text { objective function } \\ h & \text { specific enthalpy } \\ L & \text { length } \\ m & \text { mass } \\ \dot{m} & \text { mass flow rate } \\ P & \text { pressure } \\ \dot{Q} & \text { heat transfer rate } \\ S & \text { entropy } \\ S & \text { specific entropy } \\ T & \text { temperature } \\ t & \text { time } \\ u & \text { control input } \\ U A & \text { overall heat transfer coefficient } \\ V & \text { volume } \\ \nu & \text { velocity } \\ \dot{W} & \text { work transfer rate (power) } \\ X & \text { exergy } \\ \dot{X} & \text { exergy transfer rate } \\ \bar{\chi} & \text { mean quality } \\ \bar{\gamma} & \text { mean void fraction } \\ \zeta & \text { normalized zone length } \\ \eta & \text { efficiency } \\ \rho & \text { density }\end{array}$

\begin{tabular}{lll}
$\mathrm{m}^{2}$ & \multicolumn{2}{l}{ Subscript } \\
$\%$ of maximum & $a$ & air \\
$\mathrm{kW}$ & $c$ & condenser \\
$\mathrm{kJ}$ & $c 1$ & superheated fluid region in condenser \\
$\mathrm{kJ}$ & $c 2$ & two-phase fluid region in condenser \\
$\mathrm{kJ} \cdot \mathrm{kg}^{-1}$ & $c 3$ & subcooled fluid region in condenser \\
$\mathrm{m}$ & $C R$ & cross-sectional area \\
$\mathrm{kg}$ & $c v$ & control volume \\
$\mathrm{kg} \cdot \mathrm{s}^{-1}$ & $d e s t$ & destroyed \\
$\mathrm{kPa}$ & $e$ & evaporator \\
$\mathrm{kW}$ & $e 1$ & two-phase fluid region in evaporator \\
$\mathrm{kJ} \cdot \mathrm{K}^{-1}$ & $e 2$ & superheated fluid region in evaporator \\
$\mathrm{kJ} \cdot(\mathrm{kg} \cdot \mathrm{K})^{-1}$ & $g$ & gaseous state \\
$\mathrm{K}$ & $g e n$ & generation \\
$\mathrm{s}$ & $H$ & high temperature environment \\
-- & $i$ & in (inlet) \\
$\mathrm{kJ} \cdot(\mathrm{s} \cdot \mathrm{K})^{-1}$ & $k$ & compressor \\
$\mathrm{m}^{3}$ & $L$ & low temperature environment \\
$\mathrm{m} \cdot \mathrm{s}^{-1}$ & $l$ & liquid state \\
$\mathrm{kW}$ & $o$ & outlet \\
$\mathrm{kJ}$ & $r, R$ & refrigerant \\
$\mathrm{kW}$ & $v$ & EEV \\
$\mathrm{dimensionless}$ & $w$ & wall \\
dimensionless & 0 & reference environment \\
$\mathrm{dimensionless}$ & 12 & between the first and second fluid regions \\
dimensionless & 23 & between the second and third fluid regions \\
$\mathrm{kg} \cdot \mathrm{m}^{-3}$ & $I$ & first law of thermodynamics \\
& &
\end{tabular}


$\psi \quad$ specific flow exergy $\quad \mathrm{kJ} \cdot \mathrm{kg}^{-1} \quad$ II $\quad$ second law of thermodynamics

\section{ACKNOWLEDGEMENTS}

585 This work was supported in part by the Department of Energy (DOE) Office of Science Graduate Fellowship 586 Program.

\section{REFERENCES}

[1] T. J. Kotas, The Exergy Method of Thermal Plant Analysis, London: Butterworths, 1985.

[2] J. U. Ahamed, R. Saidur and H. H. Masjuki, "A review on exergy analysis of vapor compression refrigeration system," Renewable and Sustainable Energy Reviews, vol. 15, no. 3, pp. 1593-1600, 2011.

[3] M. Padilla, R. Revellin and J. Bonjour, "Exergy analysis of R413A as replacement of R12 in a domestic refrigeration system," Energy Conversion and Management, vol. 51, pp. 2195-2201, 2010.

[4] H. Mahabadipour and H. Ghaebi, "Development and comparison of two expander cycles used in refrigeration system of olefin plant based on exergy analysis," Applied Thermal Engineering, vol. 50, no. 1, pp. 771-780, 2012.

[5] A. Bejan, "Fundamentals of exergy analysis, entropy generation minimization, and the generation of flow architecture," International Journal of Energy Research, vol. 26, pp. 545-565, 2002.

[6] P. K. Nag and S. De, "Design and Operation of a Heat Recovery Steam Generator with Minimum Irreversibility," Applied Thermal Engineering, vol. 17, no. 4, pp. 385-391, 1997.

[7] J. Vargas and A. Bejan, "Thermodynamic optimization of finned crossflow heat exchangers for aircraft environmental control systems," International Journal of Heat and Fluid Flow, vol. 22, pp. 657-665, 2001.

[8] D. Liberzon, Calculus of Variations and Optimal Control Theory: A Concise Introduction, Princeton, New Jersey: Princeton University Press, 2012.

[9] Y. A. Cengel and M. A. Boles, Thermodynamics: An Engineering Approach, 6th ed., Boston: The McGrawHill Companies, Inc., 2008.

[10] M. J. Moran and H. N. Shapiro, Fundamentals of Engineering Thermodynamics, John Wiley \& Sons, Inc., 2004.

[11] A. Bejan, Advanced Engineering Thermodynamics, Hoboken, New Jersey: John Wiley \& Sons, Inc., 2006.

[12] W. Stoecker and J. Jones, Refrigeration and Air-Conditioning, New York: McGraw-Hill Book Company, 1983.

[13] B. P. Rasmussen, "Dynamic modeling for vapor compression systems - Part I: Literature Review," HVAC\&R Research, vol. 18, no. 5, pp. 934-955, 2012.

[14] S. Bendapudi and J. E. Braun, "A Review of Literature on Dynamic Models of Vapor Compression Equipment," ASHRAE Report \#4036-5, May 2002. 
[15] T. L. McKinley and A. G. Alleyne, "An advanced nonlinear switched heat exchanger model for vapor compression cycles using the moving-boundary method," International Journal of Refrigeration, vol. 31, no. 7, pp. 1253-1264, 2008.

[16] B. Li and A. G. Alleyne, "A dynamic model of a vapor compression cycle with shut-down and start-up operations," International Journal of Refrigeration, vol. 33, no. 3, pp. 538-552, 2010.

[17] K. Amano, "Heat pump apparatus and control method thereof". Europe Patent EP2469201 A3, 2012.

[18] X. D. He, S. Liu, H. H. Asada and H. Itoh, "Multivariable Control of Vapor Compression Systems," HVAC\&R Research, vol. 4, no. 3, pp. 205-230, 1998.

[19] R. Shah, B. P. Rasmussen and A. G. Alleyne, "Application of a Multivariable Adaptive Control Strategy to Automotive Air Conditioning Systems," International Journal of Adaptive Control and Signal Processing, vol. 18, no. 2, pp. 199-221, 2004.

[20] J.-L. Lin and T.-J. Yeh, "Control of multi-evaporator air-conditioning systems for flow distribution," Energy Conversion and Management, vol. 50, no. 6, pp. 1529-1541, 2009.

[21] B. P. Rasmussen and A. G. Alleyne, "Gain scheduled control of an air conditioning system using the Youla parameterization," IEEE Transactions on Control Systems Technology, vol. 18, no. 5, pp. 1216-1225, 2010.

[22] T. G. Hovgaard, L. F. Larsen, K. Edlund and J. B. Jørgensen, "Model predictive control technologies for efficient and flexible power consumption in refrigeration systems," Energy, vol. 44, no. 1, p. 105-116, 2012.

[23] M. S. Elliott and B. P. Rasmussen, "A Model-Based Predictive Supervisory Controller for Multi-Evaporator HVAC Systems," in Proceedings of the American Control Conference, 2009.

[24] L. F. S. Larsen, C. Thybo, J. Stoustrup and H. Rasmussen, "A Method for Online Steady State Energy Minimization with Application to Refrigeration Systems," in Proceedings of the 43rd IEEE Conference on Decision and Control, 2004.

[25] J. P. Koeln and A. G. Alleyne, "Decentralized Controller Analysis and Design for Multi-Evaporator Vapor Compression Systems," in Proceedings of the 2013 American Control Conference, Washington, DC, 2013.

[26] J. Maciejowski, Predictive Control with Constraints, Prentice Hall, 2000.

[27] D. Q. Mayne, J. B. Rawlings, C. V. Rao and P. O. M. Scokaert, "Constrained model predictive control: Stability and optimality," Automatica, vol. 36, pp. 789-814, 2000.

[28] N. Jain and A. G. Alleyne, "Transient Exergy Destruction Analysis for a Vapor Compression System," in International Refrigeration and Air Conditioning Conference, West Lafayette, 2014.

[29] J. H. Doty, J. A. Camberos and K. L. Yerkes, "Approximate Approach for Direct Calculation of Unsteady Entropy Generation Rate for Engineering Applications," in Proceedings of the 50th AIAA Aersopace Sciences Meeting, Nashville, 2012.

[30] B. P. Rasmussen, Control-Oriented Modeling of Transcritical Vapor Compression Systems, Urbana, IL: University of Illinois at Urbana-Champaign, M.S. Thesis, 2000. 
[31] N. Jain, Thermodynamics-Based Optimization and Control of Integrated Energy Systems, Urbana: University of Illinois at Urbana-Champaign, 2013.

[32] G. F. Franklin, J. D. Powell and M. Workman, Digital Control of Dynamic Systems, 3rd Edition ed., Half Moon Bay, CA: Ellis-Kagle Press, 1998.

[33] E. Sciubba and G. Wall, "A brief Commented History of Exergy From the Beginnings to 2004," International Journal of Thermodynamics, vol. 10, no. 1, pp. 1-26, 2007.

[34] W. L. R. Gallo and L. F. Milanez, "Choice of a Reference State for Exergetic Analysis," Energy, vol. 15, no. 2, pp. 113-121, 1990.

[35] B. P. Rasmussen, Dynamic Modeling and Advanced Control of Air Conditioning and Refrigeration Systems, Urbana, IL: University of Illinois at Urbana-Champaign, Ph.D. Thesis, 2005. 